Research Article

\title{
Game Theoretical Perspectives on Pricing Decisions in Asymmetric Competing Supply Chains
}

\author{
Tao Li, ${ }^{1}$ Qi Tan, ${ }^{2}$ and Weirong Liu $\mathbb{D}^{3}$ \\ ${ }^{1}$ Business School, Shandong University of Technology, Shandong 255000, China \\ ${ }^{2}$ School of Economics and Management, Xi'an University of Technology, Xi'an 710048, China \\ ${ }^{3}$ National Demonstration Center for Experimental Modern Circulation Education, Shanghai Business School, \\ Shanghai 201400, China \\ Correspondence should be addressed to Weirong Liu; liuwr@sbs.edu.cn
}

Received 10 December 2019; Revised 28 December 2019; Accepted 30 December 2019; Published 19 February 2020

Academic Editor: Maria Alessandra Ragusa

Copyright (C) 2020 Tao Li et al. This is an open access article distributed under the Creative Commons Attribution License, which permits unrestricted use, distribution, and reproduction in any medium, provided the original work is properly cited.

\begin{abstract}
This paper investigates a dual exclusive channel model in which each manufacturer distributes its goods through a single exclusive retailer, but two goods are substitute. The decision rule between two channels is Nash game in Case 1, while it is Stackelberg game in Case 2. From manufacturer Stackelberg (MS), retailer Stackelberg (RS), and Nash game (VN) theoretic perspectives, nine game models are developed to examine the effect of product substitutability and relative channel status on pricing decisions at both horizontal competition and vertical competition levels. The analysis suggests that the type of price leadership scenarios, the level of product substitutability, and the relative channel status play a significant role in decision making. For instance, in case 1, the symmetric leadership (two manufacturers or two retailers are leaders) is always the dominant strategy and equilibrium for either two manufacturers or two retailers regardless of product substitutability and relative channel status. Nevertheless, the asymmetric leadership may lead channel members to encounter a prisoner's dilemma if the relative channel status is small. By contrast, in Case 2 , the symmetric leadership is not the unique dominant strategy for either two manufacturers or two retailers. In contrast to many earlier results, we also show that whether the first-mover and the late-mover advantages exist, depending on the level of related channel status.
\end{abstract}

\section{Introduction}

The importance of pricing decision has been received significant attention in business and academics. It has been well known that cost and demand play a significant role in pricing decision. However, in practice, a firm's pricing decision depends not only on its cost and demand but also on its position or bargaining power in the market. In particular, numerous previous studies have shown that price leadership has a major effect on firm performance and supply chain overall efficiency [1-5] and most of these studies focus on the leadership problem at the manufacturer or retailer level. Nevertheless, in a complex and competitive business environment, the individual businesses no longer compete as stand-alone entities, but rather as supply chains (including the competition at both manufacturer and retailer levels). For instance, in the competitive market of smartphones, Microsoft and Nokia constituted a supply chain to compete with other supply chains such as Google and Samsung. To address the above issues, we study a model of a competing supply chains, where in each supply chain, a manufacturer sells its product exclusively through a downstream retailer. As such, the model exists both manufacturer and retailer level competitions. As noted by McGuire and Staelin [6], there are a number of industries which can be represented by this structure, including gasoline, automobile, soft drinks, industrial/farm heavy equipment, and retail chains with large wholesalers supporting exclusive retail outlets, among others.

Our work is related to the large volume of literature on price leadership in the past several decades. Extant studies mainly focus on three game structures, that is, MS, RS, and 
VN games, where all manufacturers and retailers share equal, or balanced, decision-making power (the symmetric leadership situation). Such supply chains are quite common in practice; in reality, Wal-Mart, Tesco, and Home Depot are usually referred as power retailers; Caterpillar and Apple are often viewed as power manufacturers, while Wal-Mart and Procter \& Gamble are almost equal in bargaining. We relax this conventional assumption to introduce the asymmetric leadership situation and the horizontal Stackelberg competition (two channels have unequal power in determining pricing decisions) to our model. It is reasonable to introduce these two factors, because supply chains are generally comprised of individual members who often have different objectives, which always lead to the asymmetric leadership situation in the real world. Moreover, a channel into the market later may have a relatively lower barging power. Specifically, there is a horizontal Stackelberg competition between the two channels. A good example is that Coca-Cola entered the Chinese market very early, while Pepsi entered late, and Pepsi had to follow the retail price of Coca-Cola. Another example is that Surface Pro 4 and iPad Pro are similar portable personal computers manufactured by Microsoft and Apple, respectively, but these two competitive electronic products were launched at different times. All these examples mean that the timing of product coming into the market has a significant effect on the performance of firms. In other words, there exist horizontal Stackelberg competitions among supply chains, the weaker supply chain makes his/her decisions only after observing the decisions of the power supply chain. Here, from the aforementioned analysis, we try to ask the following research questions:

(i) What are the joint impacts of product substitutability and asymmetric channel status on the performance of channel members and the entire supply chain under different price leadership scenarios?

(ii) What are the impacts of vertical and horizontal Stackelberg competitions on the optimal pricing decisions and the performance of channel members?

(iii) Under what conditions will the first-mover advantage and the late-mover advantage exist?

To address the above issue, this paper intends to provide a novel answer to these questions, with explicit consideration of the effects of product substitutability and relative channel status on pricing decision under different price leadership scenarios of two cases. The game rule between two channels is Nash game in Case 1 (a dual exclusive channel without horizontal Stackelberg competition), while Stackelberg game in Case 2 (a dual exclusive channel with horizontal Stackelberg competition). Meantime, this paper considers the symmetric price leadership scenario as well as the asymmetric price leadership scenario. To differ from previous works for a single channel or a monopoly common retailer/manufacturer channel, this paper indicates that the type of price leadership scenarios, the level of product substitutability, and the relative channel status play a significant role in decision making. In particular, this paper makes contributions to three aspects. First, this paper extends the current literature related to pricing decision by considering a dual exclusive channel model from both vertical and horizontal Stackelberg competitions. Second, by using the game-theoretical approach, analytical equilibrium solutions are obtained in each of the nine price leadership scenarios. Third, this paper has five main findings: (1) in Case 1, the symmetric leadership is always the dominant strategy for channel members, while the asymmetric leadership may make the channel members encounter a prisoner's dilemma; (2) in Case 1, the retailer could minimize its disadvantage of being a follower by a larger base market advantage and higher product substitutability; (3) in Case 2, the symmetric leadership is not the unique dominant strategy for either two manufacturers or two retailers; (4) in Case 2, whether the late-mover advantage and the first-mover advantage exist depends on the relative channel status; (5) whether the leadership is profitable for the whole channel depends on the level of product substitutability and the relative channel status no matter in Case 1 and Case 2.

The remainder of this study is organized as follows. We first review the related literature in Section 2. We then describe the model in section 3. Subsequently, we present the equilibrium results in Sections 4 and 5. The whole supply chain efficiency and consumers welfare are discussed to provide new managerial insight in Section 6. Lastly, we present conclusions and outlooks in Section 7, and all proofs are given in Appendix A.

\section{Literature Review}

This paper investigates the impacts of product substitutability and relative channel status on pricing competition under different price leadership scenarios at both vertical and horizontal levels. Thus, there are three streams of the literature related to this work: the first stream relates to price competition without horizontal Stackelberg competition, the second stream deals with price competition with horizontal Stackelberg competition and the third stream relates to the relative channel status. Meantime, the most related studies are presented in Table 1.

There has been an extensive literature on price competition without horizontal Stackelberg competition. We start our analysis with reviewing three common supply chain structures. The first supply chain structure related to the price leadership is a single channel. According to Seyedesfahan et al. [10], they study cooperative advertising and pricing decisions in a single-channel structure under MS, RS, $\mathrm{VN}$, and cooperation games. Their research finding indicates that the cooperation game cannot always result in the highest profit for the entire supply chain. Then, Chaeb and Rasti-Barzoki [11] also investigate cooperative advertising and pricing decisions using a similar power structure setting. Their finding is consistent with [10] but from a different perspective. Shi et al. [12] explore the influence of leadership on both channel members and the entire supply chain with uncertain demand. They suggest that whether a firm gains 
TABLE 1: Comparison of previous models with the current study.

\begin{tabular}{|c|c|c|c|c|c|c|c|c|c|}
\hline \multirow[t]{2}{*}{ Authors } & \multicolumn{3}{|c|}{ Channel structure } & \multicolumn{2}{|c|}{ Channel status } & \multicolumn{2}{|c|}{$\begin{array}{l}\text { Leadership } \\
\text { status }\end{array}$} & \multicolumn{2}{|c|}{$\begin{array}{l}\text { Leadership } \\
\text { competition }\end{array}$} \\
\hline & MCR & MCM & $\mathrm{DE}$ & SCS & ACS & SL & $\mathrm{AL}$ & WHC & $\mathrm{NHC}$ \\
\hline Choi [1] & $\sqrt{ }$ & & & $\sqrt{ }$ & & $\sqrt{ }$ & & & $\sqrt{ }$ \\
\hline Yang et al. [7] & & $\sqrt{ }$ & & & $\sqrt{ }$ & $\sqrt{ }$ & & & $\sqrt{ }$ \\
\hline Zhang et al. [8] & & & $\sqrt{ }$ & & & $\sqrt{ }$ & & & $\sqrt{ }$ \\
\hline Edirisinghe et al. [9] & $\sqrt{ }$ & & & $\sqrt{ }$ & & $\sqrt{ }$ & $\sqrt{ }$ & $\sqrt{ }$ & $\sqrt{ }$ \\
\hline Chung and Lee [3] & $\sqrt{ }$ & & & $\sqrt{ }$ & & $\sqrt{ }$ & & & $\sqrt{ }$ \\
\hline This paper & & & $\sqrt{ }$ & $\sqrt{ }$ & $\sqrt{ }$ & $\sqrt{ }$ & $\sqrt{ }$ & $\sqrt{ }$ & $\sqrt{ }$ \\
\hline
\end{tabular}

MCR represents monopoly common retailer channel, MCM represents monopoly common manufacturer channel, DE represents dual exclusive channel, SCS represents symmetric channel status, ACS represents asymmetric channel status, SL represents symmetric price leadership, AL represents asymmetric price leadership, WHC represents channel structure with horizontal Stackelberg competition, and NHC represents channel structure without horizontal Stackelberg competition.

more benefit by playing the leader's role depends on the expected demand model but not on the demand shock model. Chen and Wang [13] investigated a smartphone supply chain consisting of a handset manufacturer and a telecom service operator under MS, RS, VN, and operator Stackelberg games. They demonstrate that the channel members always benefit from playing the Stackelberg leader, while the entire supply chain is always profitable in VN game than that of other games. Chen et al. [14] examine how different price leadership scenarios (MS, RS, and VN games) influence the coordination in a sustainable supply chain under a two-part tariff. They point out that the two-part tariff contract can coordinate the sustainable supply chain under different price leadership structures. The second supply chain structure is a monopoly common retailer channel that consists of two manufacturers and a retailer. In a seminal work on price leadership, Choi [1] develops a monopoly common retailer channel model, considering linear demand and nonlinear demand under MS, RS, and VN games. $\mathrm{He}$ demonstrates that a Stackelberg leader gains more profits from its leadership but hurts the followers. Zhao et al. [15] examine the pricing decisions of a monopoly common retailer channel structure under eight pricing game models, their observation shows that the leadership between two manufacturers and a common retailer does not affect the profit of the entire supply chain under Bertrand competition or cooperation strategy. Zhao et al. [16] extend this research by examining the pricing decisions of a similar channel structure under different price leadership scenarios. The third chain structure is a monopoly common manufacturer channel; Yang et al. [7] consider the pricing and quantity decisions of a monopoly manufacturer channel model under MS game, the total profit of the duopolistic retailers who act as the followers could gain more benefits than the manufacturer's as long as the degree of dissimilarity between the duopolistic retailers' market demands is large enough. However, most of the literature listed above neglect the competition at both retailer and manufacturer levels.

From the aforementioned analysis, very limited studies have attempted to examine the competition at both manufacturer and retailer levels, and hence this paper extends these models to the fourth channel structure: a dual exclusive channel structure. Our paper is most closely related to Zhang et al. [8]; they examine three games (MS, RS, and Nash games) in a dual exclusive channel. They demonstrate that it is a dominant strategy and equilibrium for channel members to play the Stackelberg leader regardless of product substitutability and relative channel status. However, they do not consider asymmetric leadership scenarios, namely, retailer 1 (manufacturer 1) and manufacturer 2 (retailer 2) acting as the Stackelberg leader. Meantime, our paper is also closely related to Chung and Lee's study [3]. They develop a monopoly common retailer channel model, considering symmetric and asymmetric price leadership scenarios, under eight pricing game models. In reality, the channel into the market later may have a relatively lower barging power. Specifically, there is a horizontal Stackelberg competition between the two channels. However, both Zhang et al. [8] and Chung and Lee [3] do not consider the horizontal Stackelberg competition. Note that there are some papers investigating price decisions in a dual channel including a traditional channel and an online channel [17]. This paper extends the existing literature but considers the horizontal Stackelberg competition in a dual exclusive channel, which allows us to examine all the possible price leadership scenarios.

Research on horizontal pricing competition between two or more members in supply chains can be traced back to Jeuland and Shugan's study [18]. Since then, increasing literature has focused on this problem. For example, $\mathrm{Wu}$ et al. [19] investigate the pricing decisions in a noncooperative supply chain that consists of two retailers and one common supplier under six price leadership structures. Wei et al. [5] study the pricing decisions of a monopoly common retailer channel model under five games, namely, MS-Bertrand, MS-Stackelberg, RS-Bertrand, RS-Stackelberg, and VN games; they suggest that the optimal prices and the maximum profits decrease with self-price and cross-price sensitivities. Ma and Li [20] investigate Bertrand-Stackelberg pricing decisions in a monopoly common retailer channel model with uncertain demand. Huang et al. [21] consider a pricing competition and a cooperation problem in a monopoly manufacturer channel model under six decentralized game models. Edirisinghe et al. [9] develop eight game models to examine the implications of channel power on supply chain stability in a monopoly common 
retailer channel model. Luo et al. [22] develop seven game models to examine the impact of leadership scenarios on the pricing decisions and the performance of the manufacturers and the retailer. Wang et al. [23] investigate the pricing and service decisions of complementary products in a dualchannel supply chain that consists of two manufacturers and one common retailer under three game models. Nevertheless, all the above literature has not investigated the pricing decisions in a dual exclusive channel under different price leadership scenarios. Li et al. [24] investigate the effect of product substitutability and relative channel status on pricing decisions under different leadership scenarios of three channel structures. Liu et al. [25] examine the emission reduction performance for supply chain members in both single channel and exclusive dual-channel structures. However, both Li et al. [24] and Liu et al. [25] neglect the impact of Nash games on pricing decisions from a vertical competition perspective.

Another area often neglected in the previous studies is that the two channels have asymmetric base market shares. For instance, Yang et al. [7] and Edirisinghe et al. [9] explore supply chain structures under symmetric and asymmetric relative channel status. However, all of them analyze numerically the effect of relative channel status on pricing decisions under different price leadership in a monopoly common retailer channel model or a monopoly manufacturer channel. To the best of our knowledge, few papers have focused on the joint effects of product substitutability and asymmetric related channel status on the value of price leadership in a dual exclusive channel. Additionally, there are some literatures to investigate the price leadership from empirical methods, such as Kadiyali et al. [26], Kuiper et al. [27], and Costa and Pavone [28]. In general, both analytical and empirical analyses show that the pricing decision critically depends on the leader-follower relationship.

\section{The Model}

This paper considers a dual exclusive channel $[6,29-31]$ where each manufacturer sells a product exclusively through a franchised retailer but two goods are substitute, as illustrated in Figure 1. We mainly explore the impacts of asymmetric price leadership, asymmetric relative channel status, and product substitutability on the pricing decision of two cases (note that the focuses between Case 1 and Case 2 are different; we focus on the vertical Stackelberg competition in Case 1, while on the horizontal Stackelberg competition in Case 2). As shown in Figure 1(a), Case 1 is a dual exclusive channel without horizontal Stackelberg competition in which two channels are equally powerful in decisionmaking. The decision rule between the two channels is Nash game. Similarly, Case 2, as shown in Figure 1(b), is a dual exclusive channel with horizontal Stackelberg competition in which the weaker channel makes decision after the powerful channel.

To characterize demand for the dual exclusive channel models, we adopt the elegant framework by Ingene and Parry [32] and employ a utility function of a representative consumer from the perspective of aggregate demand as follows:

$$
U=\sum_{i=1}^{2}\left(A_{i} D_{i}-\frac{D_{i}^{2}}{2}\right)-\theta D_{1} D_{2}-\sum_{i=1}^{2} P_{i} D_{i},
$$

where the index $i=1,2$, represents different manufacturers, retailers, or channels. $D_{i}$ represents the demand for the product produced and sold by supply chain i. $\theta(0 \leq \theta<1)$ denotes product substitutability, $\theta=0$ implies the channels are purely monopolistic, as $\theta$ approaches 1 , the two products toward being completely substitutable. $A_{i}$ is channel $i$ 's initial base market. $p_{i}$ represents the retail price of retailer $i$, and $w_{i}$ represents the wholesale price of manufacturer $i$. It is worth noting that the utility function has since been widely utilized in the economics, marketing, and operations management literature [33-37].

Maximization of the utility function yields the demand for different channels as follows:

$$
D_{i}=\frac{A_{i}-\theta A_{3-i}-p_{i}+\theta p_{3-i}}{1-\theta^{2}} .
$$

The function form, which is more suitable to study the impact of production competition, is different from a commonly used demand function [38, 39].

To facilitate our discussion, we define, $\Omega \equiv A_{1} / A_{2}$ as the relative channel status, which will play the prime role of this research. If $\Omega>1$, channel l's initial base demand is larger than channel 2's and vice versa. If $\Omega=1$, it means that the two channels is symmetric in the relative channel status; otherwise, the two channels are asymmetric. Note that, for Case 1, there exist three possible values (such that $\Omega=1$, $\Omega>1$ and $\Omega<1$ ), while $\Omega \geq 1$ or $\Omega \leq 1$ is required for Case 2 . This is because the leader channel has the larger base demand if the follower channel is new to the market, that is $\Omega>1$. This setting can be referred to Huang et al. [21] and Edirisinghe et al.'s studies [9]. During a period of time of developing, the leader channel may have the same market share, that is, $\Omega=1$ (note that this symmetry is meaningful because it presents the most drastic competition market.)

To enable fair comparison among the various price leadership scenarios and for simplicity, without loss of generality, we assume that the production costs and the operational costs are normalized to zero, a similar setting used in Chung and Lee [3] and Karray et al. [40]. Thus, the profits of manufacturer $i$ and retailer $i$ are, respectively, represented as follows:

$$
\begin{aligned}
\max \pi_{m i}\left(w_{i}, w_{3-i}\right) & =w_{i} D_{i}, \\
\max \pi_{r i}\left(p_{i}, p_{3-i}\right) & =\left(p_{i}-w_{i}\right) D_{i} .
\end{aligned}
$$

where parameter $\pi_{m i}$ is manufacturer $i$ 's profit, $\pi_{r i}$ is retailer $i$ 's profit, $\pi_{i}$ is channel $i$ 's profit, and $\pi$ is the total profit of the entire supply chain. Furthermore, $m_{i}=p_{i}-w_{i}$ is the retail margin on product $i$.

Following the recommendations in Edirisinghe et al. [9], we also assume that a channel member is said to commit "power sacrifice" when he is willing to move a lower power position, thus foregoing the option of exercising his channel 


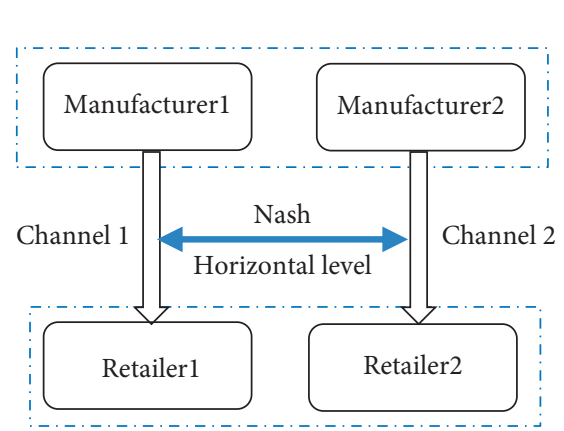

(a)

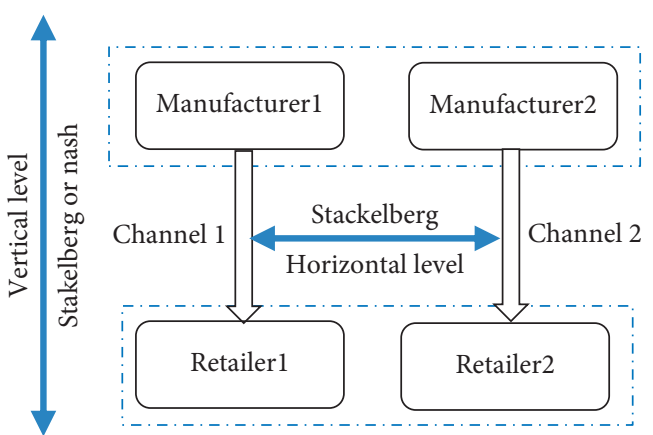

(b)

FIgURE 1: Structures of the dual channel.

power. In this regard, each member has the possibility of being a leader because the dual exclusive channel is comprised of individual members who are often guided by conflicting objective functions, which leads to nine possible price leadership scenarios (note that this assumption is very important for us to examine the notion of structure dominance and equilibrium.).

\section{Case 1: Dual Channel without Horizontal Stackelberg Competition}

As a benchmark to evaluate pricing decisions under different price leadership scenarios, we first examine a dual exclusive channel without horizontal Stackelberg competition. There are nine possible price leadership scenarios under Case 1. Three of them are symmetric and the other six are asymmetric. In all nine subgames, each proceeds as a one-stage (e.g., NVV) or two-stage game (e.g., NMM and NMV). The game rule of the symmetric price leadership is straightforward (NMM: both manufacturers are the leaders of retailers; NRR: both retailers are the leaders of manufacturers; NVV: the manufacturers and retailers are equally powerful in determining optimal channel prices), which has been used in Zhang et al. [8] and Choi [41]. Thus, the derivation process of equilibrium solutions for the symmetric price leadership scenario is omitted in the following. The decision sequence of the asymmetric price leadership scenario is as follows. To facilitate the following discussions, we use superscripts M1, $\mathrm{M} 2$, R1, and R2 to denote manufacturer 1, manufacturer 2, retailer 1 , and retailer 2 , respectively.

\section{(a) Scenario NMR}

In Scenario NMR, M1 and R2 are the Stackelberg leaders while $\mathrm{R} 1$ and $\mathrm{M} 2$ are the followers. In the first stage, M1 chooses a wholesale price using the response function of R1. Meantime, R2 chooses its margin using the response function of the M2. In the second stage, given a wholesale price and a retail price, $\mathrm{R} 1$ and $\mathrm{M} 2$ determine the retail price and the wholesale price, respectively.

(b) Scenario NMV

In Scenario NMV, M1 is the Stackelberg leader while M2 and R2 are equally powerful. M1 chooses a wholesale price using R1's reactions function in the first stage. In the second stage, given a wholesale price, $\mathrm{R} 1$ determines its retail price. In contrast, M2 and R2 make their pricing decisions simultaneously in Vertical Nash game.

(c) Scenario NRV

Scenario NRV is similar to Scenario NMV, except that R1 becomes the Stackelberg leader. R1 chooses its margin anticipating M1's reactions function in the first stage. In the second stage, M1 determines its wholesale price given the corresponding retail price. By contrast, M2 and R2 are equally powerful in determining their optimal prices in Vertical Nash game.

The decision sequence for the other three asymmetric price leadership scenarios (NRM, NVM, and NVR) can be obtained simply by switching indices 1 and 2. Furthermore, these equilibrium solutions for symmetric and asymmetric price leadership scenarios are summarized in Tables 2 and 3, respectively.

In the following subsections, we first compare the equilibrium solutions among different price leadership scenarios with symmetric relative channel status $(\Omega=1)$. Then, we examine the similar problems with the asymmetric setting $(\Omega \neq 1)$. To simplify our exposition, we organized the discussion of this analysis by comparing the equilibrium results of different price leadership scenarios for channel 1 while holding fixed the price leadership scenario for channel 2 (i.e., $\mathrm{NMj}, \mathrm{NRj} \mathrm{NVj}, \mathrm{Mj}, \mathrm{Rj}$ and $\mathrm{Vj}, j \in\{M, R, V\}$ ). This setting allows us to check whether a channel member's decision behavior affects the other one.

4.1. The Equilibrium Results with Symmetric Channel Status. Comparing the equilibrium profits of manufacturers, retailers, and channels under different price leadership scenarios gives us the following lemma. For brevity, we use PS to represent the product substitutability.

Lemma 1. Given a price leadership situation, the manufacturer 1 and the retailer 1 as a whole have the same preference from most to least favorable are given as follows,

(i) Under MS, the preference list for the manufacturer is $N M M>N M R>N M V$ and for the retailer is $N M R>N M M>N M V$ regardless $P S$ 


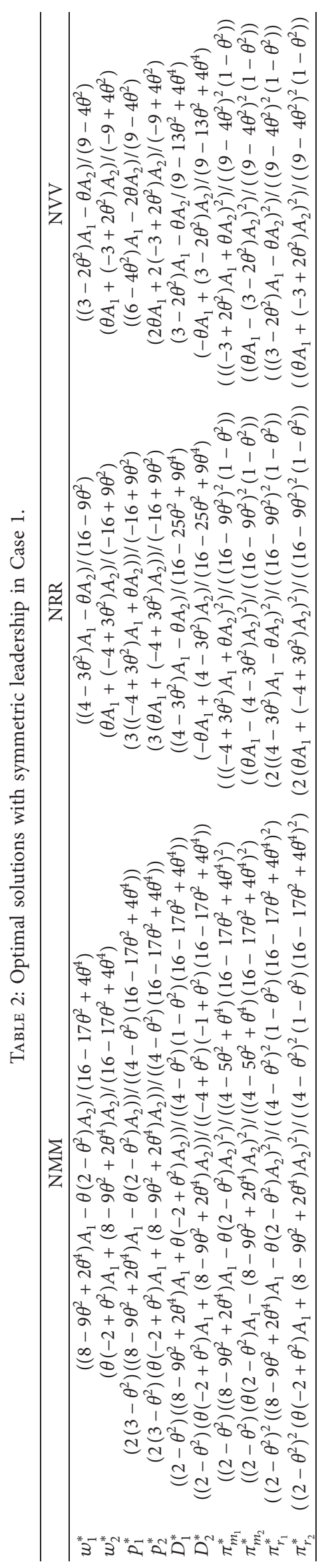




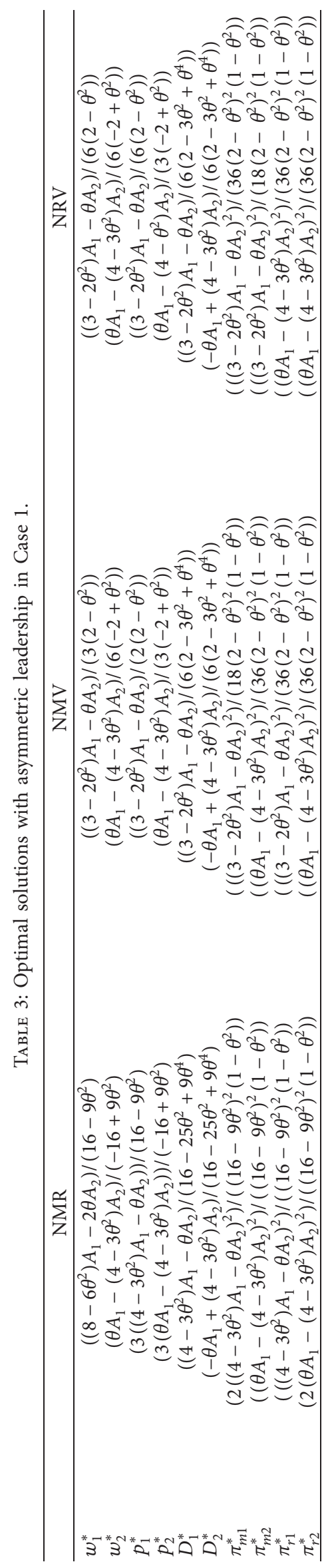


(ii) Under $R S$, the preference list is $N R R=N R M>N R V$ regardless of $P S$

(iii) Under VN, the preference list is $N V M=N V R>N V V$ regardless of $P S$

Lemma 1 is counter-intuitive that the manufacturer 1 and the retailer 1 as a whole have the same preferences by holding fixed the price leadership situation. Under MS, Scenario NMV is the least preferred by both the manufacturer 1 and the retailer 1 , while the most preferred Scenario is NMM and NMR, respectively. As $\varphi_{1}^{\mathrm{NMM}}>\varphi_{1}^{\mathrm{NMR}}>\varphi_{1}^{\mathrm{NMV}}, \varphi \in\{w, p\}$, and $D_{1}^{\mathrm{NMR}}>D_{1}^{\mathrm{NMM}}>$ $D_{1}^{\mathrm{NMV}}$, it is easy to verify that Scenario NMV results in the lowest profit for channel members due to the lowest demand and lowest price. Thus, Scenario NMV is least preferred by both the manufacturer 1 and the retailer 1 . However, it is difficult to verify that whether the most preferred Scenario is NMM. We attribute the result to the existing literature (e.g., Lee \& Staelin [4]). It is intuitive that a price leader has a positive effect on the retailer's margin but a negative effect on the demand. Moreover, the positive effect on price dominates its negative effect on demand. Thus, both the manufacturer 1 and the retailer 1 can be better off in Scenario NMM. Similar to that of MS game, we find that the least preferred Scenario is NRV/NVV and the most preferred Scenario is NRR/NRM or NVM/NVR.

We also obtain interesting results from the equilibrium profits of the manufacturer 2 and retailer 2. Specifically, these rank orders for the manufacturer 2 and retailer 2 are similar to that for the manufacturer 1 and retailer 1 in RS and $\mathrm{VN}$ games, for instance, $\pi_{m 2}^{\mathrm{NRR}}=\pi_{m 2}^{\mathrm{NRM}}>\pi_{m 2}^{\mathrm{NRV}}$ and $\pi_{r 2}^{\mathrm{NRR}}=\pi_{r 2}^{\mathrm{NRM}}>\pi_{r 2}^{\mathrm{NRV}}$. Further, Scenarios NRV and NVV is least preferred by all channel members. In contrast, under MS, we find the rank order for the manufacturer 2 is $\pi_{m 2}^{\mathrm{NMM}}>\pi_{m 2}^{\mathrm{NMV}}>\pi_{m 2}^{\mathrm{NMR}}$, while reverse for the retailer 2 . This is because a channel member is rewarded by playing the leader's role at the expense of the other channel member who becomes the follower. This result is not surprising since the leader takes advantage of the follower's reaction function and the follower simply accepts the leader's strategy to maximize his/her own profit.

Then, we investigate which structure is a relative stable equilibrium for the exclusive channel. By comparing the equilibrium profits of manufacturer 1 , retailer 1 , and channel 1 among all price leadership scenarios derives the following result. Moreover, we summarize the computation results for channel 1 of all Scenarios in Table 4 (Note that the profit for channel $i$ including manufacturer $i$ 's and retailer $i$ 's Optimal profits, which define as $\left.\pi_{i}^{*}=\pi_{m i}^{*}+\pi_{r i}^{*}\right)$.

Theorem 1. From the horizontal competition perspective (Note that to avoid confusion in concept, the concept of "horizontal Stackelberg competition" in Corollary 1 involves the leadership competition as well the horizontal interaction. The term "the horizontal interaction" is defined in terms of the direction of a channel member's reaction to the actions of its rival, while the concept of "horizontal competition" in Theorem 1 only involves the horizontal interaction.) the symmetric leadership is the dominant strategy and equilibrium for either two manufacturers or two retailers regardless of PS. However, the vertical Nash game is equilibrium for the manufacturer and the corresponding retailer from the vertical Stackelberg competition perspective. Meantime, the symmetric leadership has a positive effect on the efficiency of channels if PS is relatively high. That is,

(i) The manufacturer 1's preference list is $N M j>N V j>N R j$ regardless of $P S$, and Scenario NMM is the dominant strategy and equilibrium for the two manufacturers

(ii) The retailer 1 's preference list is $N R j>N V j>N M j$ regardless of PS, and Scenario NRR is the dominant strategy and equilibrium for the two retailers

(iii) The channel 1's preference list is NVj>NRjor NMj for $\theta \in(0,0.59)$, and Scenario NVV is the equilibrium for the two channels although it is not the dominant strategy

Here, $j \in\{M, R, V\}$.

From the proof of Theorem 1, we obtain equilibrium results for the manufacturer 1: $\pi_{m 1}^{\mathrm{NMM}}>\pi_{m 1}^{\mathrm{NMR}}>\pi_{m 1}^{\mathrm{NMV}}>\pi_{m 1}^{\mathrm{NVM}}$ $\left(\pi_{m 1}^{\mathrm{NVR}}\right)>\pi_{m 1}^{\mathrm{NVV}}>\pi_{m 1}^{\mathrm{NRR}}\left(\pi_{m 1}^{\mathrm{NRM}}\right)>\pi_{m 1}^{\mathrm{NRV}}$ for any $\theta \in(0,1)$. In contrast, we also get equilibrium results for the retailer 1 : $\pi_{r 1}^{\mathrm{NRR}}\left(\pi_{r 1}^{\mathrm{NRM}}\right)>\pi_{r 1}^{\mathrm{NRV}}>\pi_{r 1}^{\mathrm{NVM}}\left(\pi_{r 1}^{\mathrm{NVR}}\right)>\pi_{r 1}^{\mathrm{NVV}}>\pi_{r 1}^{\mathrm{NMR}}>\pi_{r 1}^{\mathrm{NMM}}$ $>\pi_{r 1}^{\mathrm{NMV}}$ for any $\theta \in(0,1)$. These rank orders show that the manufacturer 1 profit is higher in MS game, middle in VN game, and lower in RS game, and the result is reverse for the retailer 1 ; thus, the manufacturer 1 and the retailer 1 preference list is $\mathrm{NMj}>\mathrm{NVj}>\mathrm{NRj}$ and $\mathrm{NRj}>\mathrm{NVj}>\mathrm{NMj}$, $j \in\{M, V, R\}$, respectively. Similarly, the channel 1 preference list can be obtained from Table 4. Furthermore, Table 4 gives us some interesting results. When the product substitutability is lower (e.g., $\theta \in(0,0.59)$ ), the channel 1 always performs better in the $\mathrm{VN}$ game, which is consistent with Choi [41]. This is because of the negative effect of the follower's loss on profit dominates its positive effect of the leader's gain. As the product substitutability grows, such that $\theta \in[0.82,1)$, double marginalization is reduced by the intensified supply chain competition stimulated by the leadership. Thus, the channel 1 can be better off in MS game or RS game. Moreover, we find that Scenario NMV (NRV) always results in the lowest profits for both channel members and the channel 1 when the product substitutability is relatively small. Interestingly, Scenario NMV (NRV) could make the channel 1 better off if product substitutability is sufficiently intense (e.g., $\pi_{1}^{\mathrm{NMV}} \geq \pi_{1}^{\mathrm{NVV}}$ for $\theta \in[0.94,1)$ ).

Theorem 1 differs from that of Zhang et al. [8]. This is because they only consider the symmetric price leadership game scenarios (e.g., NMM, NRR, and NVV games). When the asymmetric price leadership game scenarios are considered, we obtain interesting results that are new to the literature. For instance, from the horizontal competition perspective, we find that Scenario NMM is the dominant strategy and equilibrium for the two manufacturers, while Scenario NRR is the dominant strategy and equilibrium for the two retailers. Intuitively, the manufacturer 1 (2) or retailer 1 (2) can benefit from their own leadership, and both 
TABLE 4: Rank ordering with respect to channel 1 efficiency given $\Omega=1$.

\begin{tabular}{lccccccc}
\hline$\theta$ & $(0,0.59)$ & {$[0.59,0.61)$} & {$[0.61,0.68)$} & {$[0.68,0.82)$} & {$[0.82,0.88)$} & {$[0.88,0.94)$} & {$[0.94,1)$} \\
\hline NVM & 1 & 1 & 1 & 1 & 4 & 2 & 3 \\
NVV & 2 & 3 & 4 & 3 & 4 & 4 \\
NRR & 3 & 2 & 2 & 2 & 3 & 2 & 2 \\
NMM & 4 & 4 & 5 & 5 & 5 & 1 & 5 \\
NMV & 5 & 5 & 5 & 5 & 5 & 5 \\
\hline
\end{tabular}

$\mathrm{NVM}=\mathrm{NVR}, \mathrm{NMR}=\mathrm{NRR}=\mathrm{NRM}$, and $\mathrm{NMV}=\mathrm{NRV}$.

members have necessarily an incentive to play the leader's role if all members are the possible leaderships (e.g., $\pi_{m 1}^{\mathrm{NMj}}>\pi_{m 1}^{\mathrm{NVj}}>\pi_{m 1}^{\mathrm{NRj}}$ and $\left.\pi_{m 2}^{\mathrm{NjM}}>\pi_{m 2}^{\mathrm{NjV}}>\pi_{m 2}^{\mathrm{NjR}}\right)$. Thus, the asymmetric leadership structure is not stable. In other words, the asymmetric leadership is never the equilibrium, while Scenario NMM or NRR is always the equilibrium from the horizontal competition perspective. On the other hand, the vertical Nash game is equilibrium for the manufacturer and the corresponding retailer from the vertical Stackelberg competition perspective. Interested readers can be referred to Choi [1], Mcguire and Staelin [6] and Choi [41] for an insightful discussion on the vertical Stackelberg competition. Theorem 1 also suggests that the channel equilibrium depends on the levels of product substitutability. In other words, both channels benefit from the channel absence of leaders when product substitutability is low. Hence, it is the equilibrium for both channels to choose $\mathrm{VN}$ game. It is worth noting that the symmetric vertical Nash structure is not a very stable leadership structure. However, VN game could make the channels worse off if the product substitutability is sufficiently intense. Therefore, the equilibrium becomes Scenario NMM or NRR.

4.2. The Equilibrium Results with Asymmetric Channel Status. In the previous subsection, we have discussed equilibrium solutions among different price leadership scenarios under symmetric related channel status. A question arises: does the asymmetric related channel status affect the equilibrium results for all members, even the whole channel? We are now positioned to compare these equilibrium solutions under asymmetric relative channel status. In order to have a meaningful comparison among different power structures, the common lower and upper bounds of the constrained areas are defined by $\Omega_{1}$ and $\Omega_{u}$ respectively, which can be derived from the nonnegative demand constraints: $\Omega_{l}=\theta / 3-2 \theta^{2}, \Omega_{u}=3-2 \theta^{2} / \theta$. Due to the complexity of all boundary values, we use $\widehat{\Omega}$ to denote the boundary values with various superscript and subscript combinations. Moreover, to facilitate our discussion, we use AS to denote the asymmetric related channel status.

Given a price leadership situation, then comparing among the three leadership structures give us the following lemma.

Lemma 2. Given a price leadership situation, the manufacturer 1 and the retailer 1 have the same preference under RS game or VS game, while the preference is different under MS game. That is,

(i) Under MS, the manufacturer 1's preference list is $N M M>N M R>N M V$ regardless of $P S$ and $A S$, while the preference list for retailer 1 is $N M R \geq$ $N M M \geq N M V$ if $\widehat{\Omega}_{r 1-1}^{N M M-N M R} \leq \Omega \leq \widehat{\Omega}_{r 1-1}^{N M M-N M V}$, regardless of $P S$

(ii) Under RS, the preference list is $N R R=N R M>N R V$ regardless of $P S$ and $A S$

(iii) Under VN, the preference list is NVM $=N V R>N V V$ regardless of $P S$ and $A S$

$$
\text { Here } \widehat{\Omega}_{r 1-1}^{N M M-N M R}=\theta\left(16-19 \theta^{2}+5 \theta^{4}\right) / 32-58 \theta^{2}+34 \theta^{4}
$$

$-6 \theta^{6}$ and $\widehat{\Omega}_{r 1-1}^{N M M-N M V}=16-12 \theta^{2}-3 \theta^{4}+2 \theta^{6} / 28 \theta-45 \theta^{3}+$ $24 \theta^{5}-4 \theta^{7}$.

Lemma 2 is a special case of Lemma 1. Specifically, when $\Omega=1$, Lemma 2 degenerates into Lemma 1. Similar to the symmetric relative channel status scenario, Lemma 2 shows that the manufacturer 1 and the retailer 1 have the same preferences by holding fixed the price leadership situation under RS game and VN game. This result shows that the asymmetric relative channel status has no effect on the decision behaviors of all channel members under RS and VN games. Nevertheless, under MS game, as shown in Figure 2, when the relative channel status is small (e.g., $\Omega_{1}<\Omega \leq \widehat{\Omega}_{r 1-1}^{\mathrm{NMM}-\mathrm{NMR}}$ ), the manufacturer 1 and the retailer 1 have the same preference sequence, which is consistent with Lemma 1 . If the relative channel status increases such that $\widehat{\Omega}_{r 1-1}^{\mathrm{NMM}-\mathrm{NMR}} \leq \Omega<\widehat{\Omega}_{r 1-1}^{\mathrm{NMM}-\mathrm{NMV}}$, the preference sequence for the retailer 1 is $\mathrm{NMR} \geq \mathrm{NMM}>\mathrm{NMV}$. As the relative channel status continue to grow such that $\widehat{\Omega}_{r 1-1}^{\mathrm{NMM}-\mathrm{NMV}} \leq \Omega<\Omega_{u}$, the preference sequence for the retailer 1 becomes $\mathrm{NMR}>\mathrm{NMV} \geq \mathrm{NMM}$. This observation suggests that the asymmetric relative channel status has impacts on the decision making of channel members, which is sharply in contrast with Zhang et al. [8]. They point out that the asymmetric relative channel power does not affect the decision behaviors of the channel members; this is because they do not consider the asymmetric leadership situation.

Similarly, we also explore the same properties for channel 2, the rank orders for the manufacturer 2 are $\pi_{m 2}^{\mathrm{NMM}}>\pi_{m 2}^{\mathrm{NMV}}>\pi_{m 2}^{\mathrm{NMR}}, \quad \pi_{m 2}^{\mathrm{NRM}}>\pi_{m 2}^{\mathrm{NRV}}>\pi_{m 2}^{\mathrm{NRR}} \quad$ and $\pi_{m 2}^{\mathrm{NVM}}>\pi_{m 2}^{\mathrm{NVV}}>\pi_{m 2}^{\mathrm{NVR}}$ regardless of the asymmetric channel status and the product substitutability, whereas as a whole the rank orders are reverse for the retailer 2. It is straightforward that both manufacturer 2 and retailer 2 obtain more benefits from playing the leader's role but hurts the followers. However, it is somewhat counter-intuitive that the retailer 2 obtains more benefits in Scenario NMM than that in Scenario NMV if $\widehat{\Omega}_{r 2-2}^{\mathrm{NMM}-\mathrm{NMV}} \leq \Omega<\Omega_{u}$, as shown in Figure 3, which means that the retailer 2 with the larger base 
market can be better off even if dominated by the manufacturer. This result is new to the existing literature.

Finally, we also investigate the joint effects of the channel substitutability and asymmetric channel status on the efficiency of the whole channel. Under MS game, the preference list is NMM $>$ NMR $>$ NMV when relative channel status is small (e.g., $\Omega_{1}<\Omega<\widehat{\Omega}_{1-1}^{\mathrm{NMM}-\mathrm{NMR}}$ ). As asymmetric channel status increases such that $\widehat{\Omega}_{1-1}^{\mathrm{NMM-NMR}} \leq \Omega<\widehat{\Omega}_{1-1}^{\mathrm{NMM}-\mathrm{NMV}}$, Scenario NMR will dominate Scenario NMM; thus, the preference is $\mathrm{NMR} \geq \mathrm{NMM}>\mathrm{NMV}$. Interestingly if the relative channel status is relatively high such that $\Omega_{1-1}^{N M M-1}$ $\leq \Omega<\Omega_{u}$, the new preference becomes NMR $>$ NMV $\geq$ NMM. Figure 4 graphically illustrates the phenomenon. Under RS or VN game, the preference list is similar to that of symmetric channel status. As for channel 2, most of the results are similar to the symmetric channel status case.

Similar to Theorem 1, comparing the equilibrium profits of manufacturer 1 , retailer 1 , and channel 1 of all price leadership scenarios provides us the following proposition.

\section{Proposition 1}

(i) The manufacturer 1's preference list is $N M M>N M R>N V M>N R R \quad \geq N M V>N V V>$ $N R V$ if $\Omega_{l}<\Omega \leq \widehat{\Omega}_{m 1-1}^{N M V-N R R}$ and is NMM $>N M R>$ $N M V \geq N V M>N V V>N R R>N R V$ if $\widehat{\Omega}_{m 1-1}^{N M V-N V M}$ $\leq \Omega<\Omega_{u}$

(ii) The retailer 1's preference list is $N R R>N V M>N M R \geq N M M>N R V>N V V>N M V$ if $\Omega_{l}<\Omega \leq \widehat{\Omega}_{r 1-1}^{N M M-N M R}$ and is NRR $>N R V \geq N V M>$ $N V V>N M M>N M R>N M V$ if $\widehat{\Omega}_{r 1-1}^{N R V-N V M} \leq \Omega<\Omega_{u}$

Here, NRR $=N R M, N V M=N V R, \widehat{\Omega}_{m 1-1}^{N M V-N R R}=480 \theta-$ $872 \theta^{3}+531 \theta^{5}-108 \theta^{7}+3 \sqrt{2} \theta\left(32-66 \theta^{2}+43 \theta^{4}-9 \theta^{6}\right) /$ $1152-2784 \theta^{2}+2545 \theta^{4}-1044 \theta^{6}+162 \theta^{8}, \quad \widehat{\Omega}_{r 1-1}^{N M M-N M R}=\theta$ $\left(16-19 \theta^{2}+5 \theta^{4}\right) / 32-58 \theta^{2}+34 \theta^{4}-6 \theta^{6}$ and $\widehat{\Omega}_{m 1-1}^{N M V-N V M}=$ $\widehat{\Omega}_{r 1-1}^{N R V-N V R}=2 \theta-\theta^{3}+\sqrt{2} \theta\left(1-\theta^{2}\right) / 2-\theta^{4}$.

Proposition 1 is similar to the results of symmetric channel status case, it also shows that Scenario NMM/NMR (NRR/NRM) always results in higher profit for the manufacturer 1 (retailer 1) regardless of the asymmetric relative channel status and the product substitutability. Although the asymmetric price leadership (NMR/NRM) can be profitable for a channel member, the asymmetric price leadership is not the equilibrium for either the two manufacturers or the two retailers. Furthermore, the symmetric price leadership always results in the highest profits for channel members. Thus, the symmetric leadership is the dominant strategy and equilibrium for both channel members. But Proposition 1 also shows an interesting result when the asymmetric setting is introduced to the model. If the relative channel status is small, the asymmetric leadership may not necessarily guarantee more benefits to either the manufacturer 1 or the retailer 1 when the competing channel is absent of a leader and the related channel status is sufficiently small (e.g., $\pi_{m 1}^{\mathrm{NVM}}>\pi_{m 1}^{\mathrm{NRM}} \geq \pi_{m 1}^{\mathrm{NMV}}$ for $\Omega_{1}<\Omega \leq \widehat{\Omega}_{m 1-1}^{\mathrm{NMV}-\mathrm{NRR}}$ and $\pi_{r 1}^{\mathrm{NVM}}>\pi_{r 1}^{\mathrm{NMR}}>\pi_{r 1}^{\mathrm{NRV}}$ for

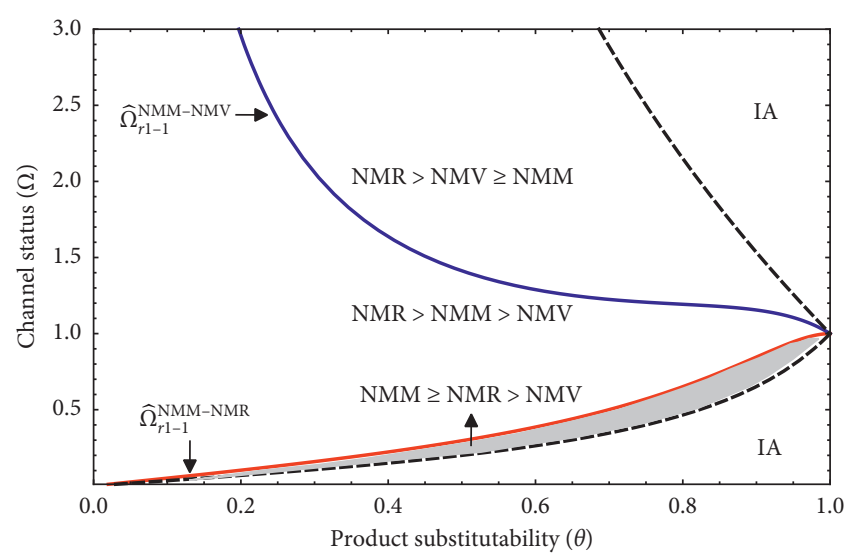

Figure 2: Comparison of retailer 1 profits under MS.

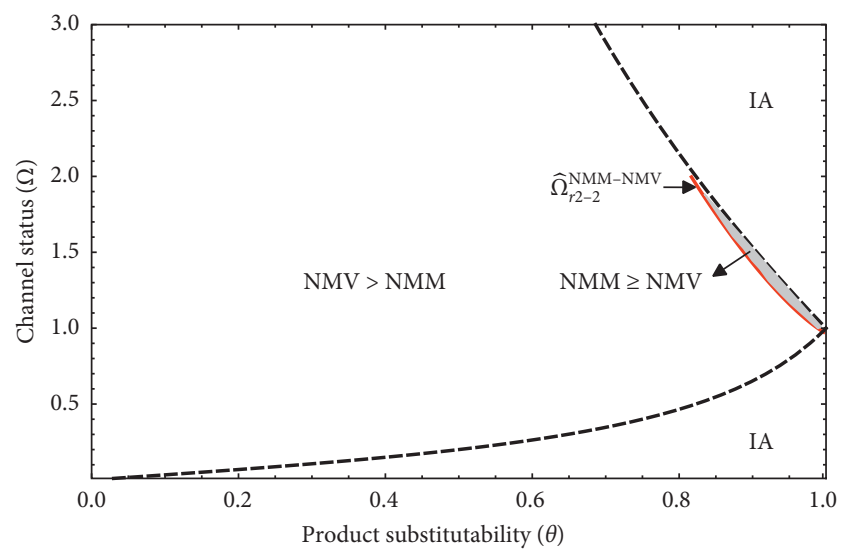

Figure 3: Comparison of retailer 2 profits under MS.

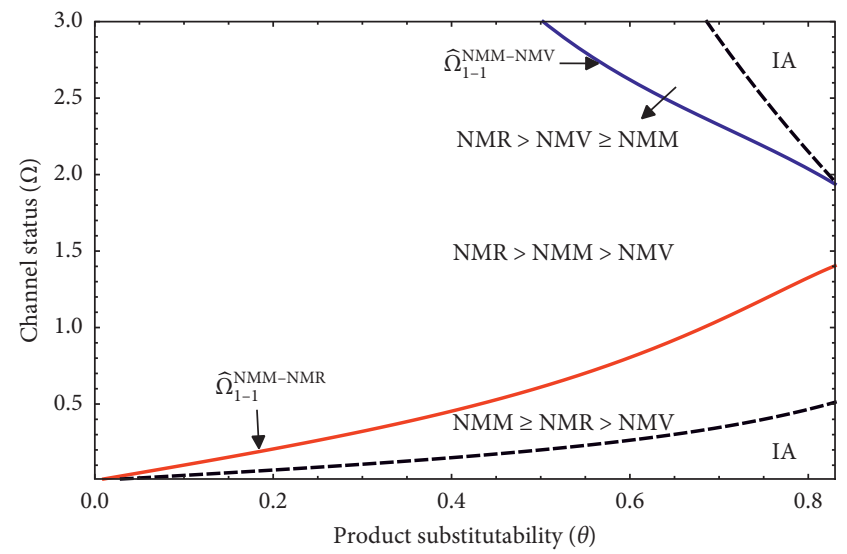

Figure 4: Comparison of channel 1 profits under MS.

$\Omega_{1}<\Omega \leq \widehat{\Omega}_{r 1-1}^{\text {NMM-NMR }}$, which is sharply in contrast with the previous studies. We should note that Chung and Lee [3] find that being a price leader is not always profitable for the retailer, but they suggest that the two manufacturers always gain more benefits from their leadership. The practical implication for the managers is that they should not only consider their own channel leadership situation but also the effect of the competing channel price leadership situation as well as the asymmetric relative channel status. 
For the channel 1, we summarize all ranker orders in Table 5 due to many threshold values for the ranker orders. As shown in Table 5, we find some interesting result that Scenario NMM could make the channel get the best performance if the relative channel status is small (e.g., $\Omega_{1}<\Omega \leq \widehat{\Omega}_{1-1}^{\mathrm{NVM}-\mathrm{NMM}}$ ), but Scenario NRR never leads the channel gain the best performance though it could result in higher profit under certain conditions. However, if the asymmetric channel status is relative high (e.g., $\widehat{\Omega}_{1-1}^{\mathrm{NMV}-\mathrm{NMM}} \leq \Omega<\Omega_{u}$ ), the channel will get the worst performance in Scenario NMM. Similarly, we summarize the core insights in Theorem 2.

Theorem 2. From the horizontal competition perspective, the symmetric leadership is always the dominant strategy and equilibrium for either two manufacturers or two retailers regardless of the asymmetric related channel status and the product substitutability. Nevertheless, the asymmetric leadership may make the channel members encounter a prisoner's dilemma if the relative channel status is small. Moreover, the symmetric leadership could have a positive effect on the efficiency of channel 1 when the asymmetric relative channel status is sufficiently low (e.g., $\Omega_{l}<\Omega<\widehat{\Omega}_{1-1}^{N V R-N M M}$ ).

Theorem 2 is similar to Theorem 1, but it depicts the joint effects of the product substitutability and asymmetric channel status on the decision behaviors of all members. In particular, the asymmetric leadership may not necessarily guarantee more benefits for the leader than the follower if the relative channel status is small (e.g., $\pi_{m 1}^{\mathrm{NVM}}>\pi_{m 1}^{\mathrm{NMV}}$ for $\Omega_{1}<\Omega \leq \widehat{\Omega}_{m 1-1}^{\mathrm{NMV}-\mathrm{NRR}}$ and $\pi_{m 2}^{\mathrm{NMV}}>\pi_{m 2}^{\mathrm{NVM}}$ for $\Omega_{1}<\Omega \leq$ $\widehat{\Omega}_{m 2-1}^{\mathrm{NVM}-\mathrm{NRR}}$ ), which implies that both the channel member and its rival prefer that neither of them play the leader's role, but if either party does, then the other has incentive to be a leader. Thus, the leaders encounter a prisoner's dilemma.

\section{Case 2: Dual Channel with Horizontal Stackelberg Competition}

Since the product substitutability and asymmetric channel status have an effect on the decision behaviors of all members under Case1, a question arises whether this finding applies to Case 2? To address this issue, we investigate by next considering a dual exclusive channel of a decision sequence, namely, the channel 1 acts as the Stackelberg leader and the channel 2 acts as the Stackelberg follower. This assumption is reasonable in the real world, which can be referred to Edirisinghe et al. [9]. Since the follower channel has the lower base demand if it is new to the market, that is $\Omega>1$. During a period of time of developing, the leader channel may have the same market share, that is $\Omega=1$. Therefore, the constrains, $\Omega \geq 1$, must be given in the following analysis.

Similar to Case 1, Case 2 also contains nine price leadership scenarios. In contrast, each subgame proceeds as a two-stage, three-stage, or four-stage game in Case 2. The game rule of the symmetric price leadership is straightforward: MM, both manufacturers are the leaders of retailers, but M1 is powerful than M2, in other words, M1 is the leader of all other members;
RR, R1 is the leader of all other members; VV, M1 (M2) and R1 (R2) are equally powerful in making decision, and M1 (R1) is more powerful than M2 (R2). Similarly, the equilibrium solutions with symmetric and asymmetric price leadership scenarios are summarized in Tables 6 and 7, respectively.

(a) MR: in this scenario, M1 is the leader of R1 as well as the leader of M2 and R2. Therefore, M1 announces a wholesale price using the response function of $\mathrm{R} 1$ who is already using the response function of R2 in the first stage. In the second stage, $\mathrm{R} 1$ sets up a retail price condition on the wholesale price of M1 as well as the response function of R2. Moreover, R2 is already using the reaction function of $\mathrm{M} 2$ to set its retail price in the third stage. In the fourth stage, given a retail price, M2 determines its wholesale price.

(b) MV: in this scenario, M1 is the leader of the other channel members, whereas neither M2 nor R2 is the leader. In the first stage, M1 announces a wholesale price using the response function of the R1. R1 decides its retail price using the response function of R2 in the second stage. In the third stage, M2 and R2 simultaneously make the pricing decisions.

(c) RV: in this scenario, R1 is the leader of the other channel members while M2 and R2 are equally powerful. In the first stage, $\mathrm{R} 1$ chooses a retail price using M1's response function. M1 decides its wholesale price using R2's response function in the second stage. In the third stage, M2 and R2 simultaneously determine their optimal prices.

We first investigate optimal solutions under the asymmetric related channel status, which is defined as $\Omega>1$. Comparing the corresponding profits among different price leadership scenarios, we obtain the following result.

Lemma 3. Given a price leadership situation, manufacturer 1 , retailer 1 , and channel 1 have the same preference from most to least favorable are given as follows. There exist only one threshold value $\widehat{\Omega}_{m 1-1}^{M M-M V}$, such that,

(i) Under $M S$, the preference list is $M M=M R \geq M V$ if $1<\Omega \leq \widehat{\Omega}_{m 1-1}^{M M-M V}$, otherwise, the preference list is $M V \geq M M=M R$

(ii) Under $R S$, the preference list is $R R=R M \geq R V$ if $1<\Omega \leq \widehat{\Omega}_{m 1-1}^{M M-M V}$, otherwise, the preference list is $R V \geq R R=R M$

(iii) Under $V N$, the preference list is $V M=V R \geq V V$ if $1<\Omega \leq \widehat{\Omega}_{m 1-1}^{M M-M V}$, otherwise, the preference list is $V V \geq V M=V R$.

$$
\begin{aligned}
\text { Here, } \quad \widehat{\Omega}_{m 1-1}^{M M-M V}=48-20 \theta^{2}-48 \theta^{4}+27 \theta^{6}+12(2-3 \theta \\
\left.2+\theta^{4}\right) \sqrt{\left(4-3 \theta^{2}\right) / 240 \theta-500 \theta^{3}+348 \theta^{5}-81 \theta^{7} .}
\end{aligned}
$$

The result for Lemma 3 is sharply in contrast with Lemma 1 and Lemma 2 in which Scenario NMV or Scenario $\mathrm{NRV}$ is least preferred by both the manufacturer 1 and retailer 1 regardless of the level of asymmetric relative 
TABLE 5: Rank ordering with respect to channel 1 efficiency given $\Omega \neq 1$ and $\theta \in(0,0.83]$.

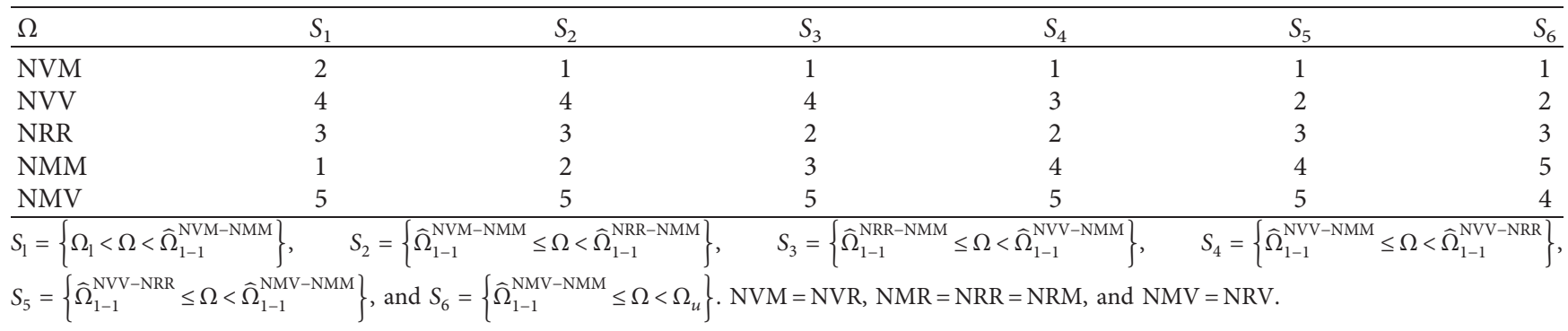

channel status and product substitutability. However, Lemma 3 shows that Scenario MV or RV could make both the channel members and the channel performer better if the related channel status is high (e.g., $\pi_{m 1}^{\mathrm{MV}} \geq \pi_{m 1}^{\mathrm{MM}}$ and $\pi_{r 1}^{\mathrm{RV}} \geq \pi_{r 1}^{\mathrm{RR}}$ if $\widehat{\Omega}_{m 1-1}^{\mathrm{MM}-\mathrm{MV}} \leq \Omega<\Omega_{u}$ ). This is because the game rule between two channels is Stackelberg game in Case 2 but is Nash game in Case 1. Furthermore, if each channel is dominated by a channel member or the channel 1 is dominated by no one and the channel 2 is dominated by a channel member, the efficiency of both channel members and the channel remains the same (e.g., $M M=M R$ and VM=VR). Finally, Lemma 3 also indicates that the preference lists of both the channel members and the channel are affected by the leadership situation as well as the level of asymmetric relative channel status. Figure 5 graphically illustrates Lemma 3. When the relative channel status is small (e.g., $1<\Omega \leq \widehat{\Omega}_{m 1-1}^{M M-M V}$ ), the manufacturer 1's preference sequence is $M M=M R \geq M V$. As the product substitutability grows, the relative channel status becomes more asymmetric, Scenario MV starts to dominate the other scenarios if the relative channel status is small is large (e.g., $\widehat{\Omega}_{m 1-1}^{\mathrm{MM}-\mathrm{MV}}<\Omega<\Omega_{u}$ ), and the new preference list is given as $\mathrm{MV}>\mathrm{MM}=\mathrm{MR}$.

Similarly, we provide comparisons for channel 2. Under MS and RS games, the rank orders are identical. That is, for the manufacturer 2, it is $\pi_{m 2}^{\mathrm{kM}}>\pi_{m 2}^{\mathrm{kV}}>\pi_{m 2}^{\mathrm{kR}}$ if $1<\Omega<\widehat{\Omega}_{m 2-2}^{\mathrm{MV}}$; otherwise, is $\pi_{m 2}^{\mathrm{kM}}>\pi_{m 2}^{\mathrm{kR}}>\pi_{m 2}^{\mathrm{kV}}, k \in\{M, R, V\}$, while the rank order is reverse for the retailer 2 . This result shows that, on the whole, being a leader is profitable for a channel member. We also illustrate this result in Figure 6; the manufacturer 2 prefers to be a follower if the relative channel status is larger, such that $\widehat{\Omega}_{m 2-2}^{\mathrm{MV}-\mathrm{MR}} \leq \Omega<\Omega_{u}$. This result is similar to Lemma 2 but is new to the previous studies $[3,9]$.

We now turn our attention to the optimal profits in all scenarios, given $\Omega>1$. Similarly, we compare the optimal profits among different price leadership scenarios and obtain the following results.

Theorem 3. The symmetric leadership is not the unique dominant strategy for either two manufacturers or two retailers. Meantime, the leadership has a positive effect on the efficiency of channel 1 if the related channel status is low. That is,

(i) The manufacturer 1's preference list is $M j>V j>R j$ regardless of $P S$, and Scenario MM is not the unique dominant strategy

(ii) The retailer 1's preference is $R j>V j>M j$ regardless of $P S$, and Scenario RR is not the unique dominant strategy (iii) The channel 1' preference list is $V j>R j$ or $M j$ if $\widehat{\Omega}_{1-1}^{V V-R R} \leq \Omega \leq \widehat{\Omega}_{1-1}^{M V-R R}$, and Scenario $V V$ is the equilibrium although it is not the dominant strategy

$$
\begin{aligned}
& \text { Here } j \in\{M, V, R\}, \quad \widehat{\Omega}_{1-1}^{V V-R R}=564 \theta-799 \theta^{3}+ \\
& 282 \theta^{5}+72 \theta\left(1-\theta^{2}\right) \sqrt{2\left(12-17 \theta^{2}+6 \theta^{4}\right)} / 720-1176 \theta^{2}+ \\
& 581 \theta^{4}-78 \theta^{6} \quad \text { and } \quad \widehat{\Omega}_{1-1}^{M V-R R}=12-17 \theta^{2}+6 \theta^{4}+ \\
& 2\left(1-\theta^{2}\right) \sqrt{3\left(12-17 \theta^{2}+6 \theta^{4}\right)} / 12 \theta-17 \theta^{3}+6 \theta^{5} .
\end{aligned}
$$

Theorem 3 is similar to Theorem 1 and Theorem 2, and it also shows that being a Stackelberg leader always results in a higher profit regardless of the rival's price leadership situation; thus, a similar explanation applies. Nevertheless, Theorem 3 shows the symmetric leadership is not the unique dominant strategy for either two manufacturers or two retailers. It also suggests that the leadership is not always bad for the performance of channels. This result is different from Theorem 1 and Theorem 2. If only consider the two Stackelberg games, the channel 1 will be indifferent to who is the leader (e.g., $\pi_{1}^{\mathrm{MM}}=\pi_{1}^{\mathrm{RR}}=\pi_{1}^{\mathrm{RM}}=\pi_{1}^{\mathrm{MR}}$ regardless of PS). As depicted in Figure 7, the leadership has a positive effect on the efficiency of channel 1 if the related channel status is low (e.g., $\pi_{1}^{\mathrm{RR}} \geq \pi_{1}^{\mathrm{VV}}>\pi_{1}^{\mathrm{MV}}$ if $1<\Omega \leq \widehat{\Omega}_{1-1}^{\mathrm{VV}-\mathrm{RR}}$ ). As the relative channel status becomes large, the leadership will have a negative effect on the performance of channel 1 .

As channel 2 is dominated by channel 1, which motivates us to ask the following research question: whether the leader channel could be always better off than the follower channel? Without loss of generality, we provide comparisons between the leader channel and the follower channel only under the symmetric leadership (e.g., MM, RR, and VV games).

Corollary 1. From the horizontal Stackelberg competition perspective, the follower channel can be better off when the channel status is relatively small. That is, channel 2 performs better than channel 1 if $1<\Omega<\widehat{\Omega}_{1-2}^{J}$, where $J \in\{M M-M M, R R-R R, V V-V V\}$.

Corollary 1 is counter-intuitive; the follower's profit may be larger than that of the leader's if the channel status is relatively small regardless of the price leadership scenarios, which implies that the first-mover advantage no longer exists if the channel status is relatively small. This result is consistent with Choi's study [41] but from a different perspective. He states that the follower could gain more benefits than that of the leader if products are highly differentiated and stores are less 


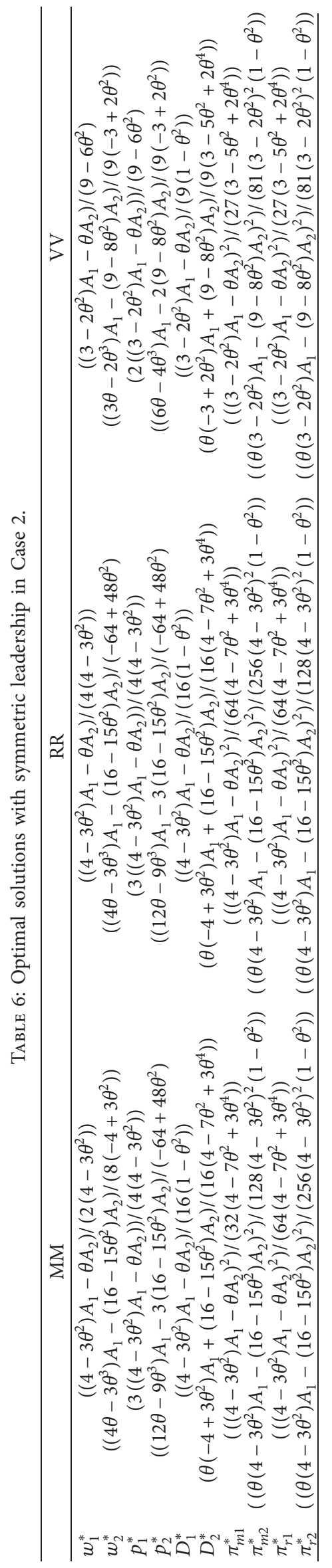




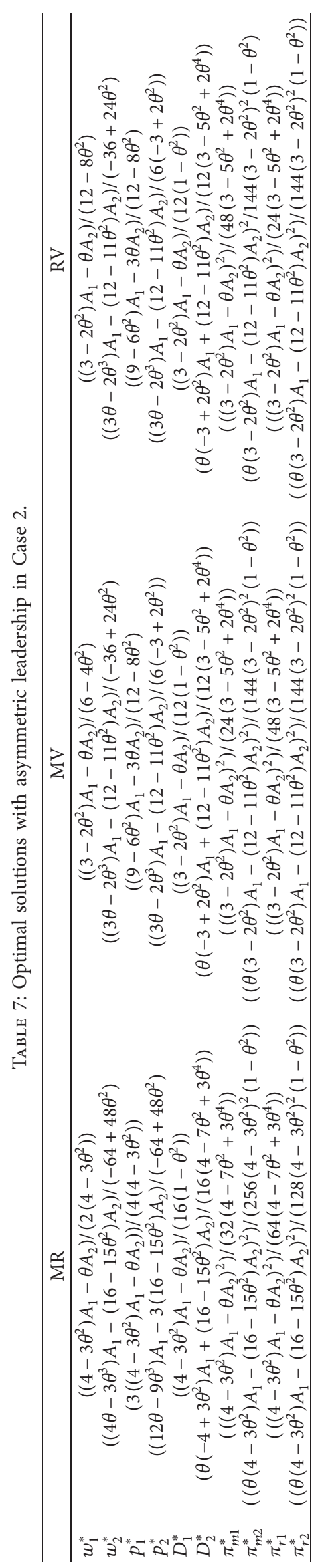




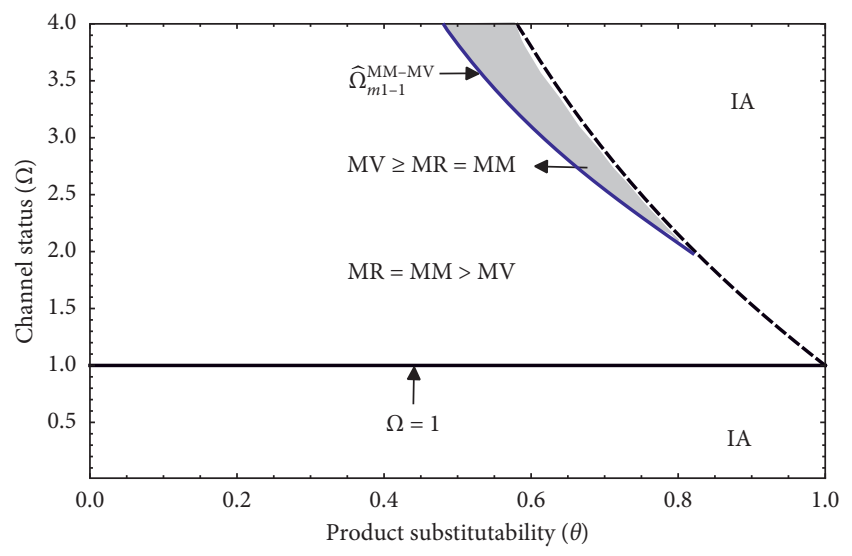

Figure 5: Comparison of manufacturer 1 profits under MS.

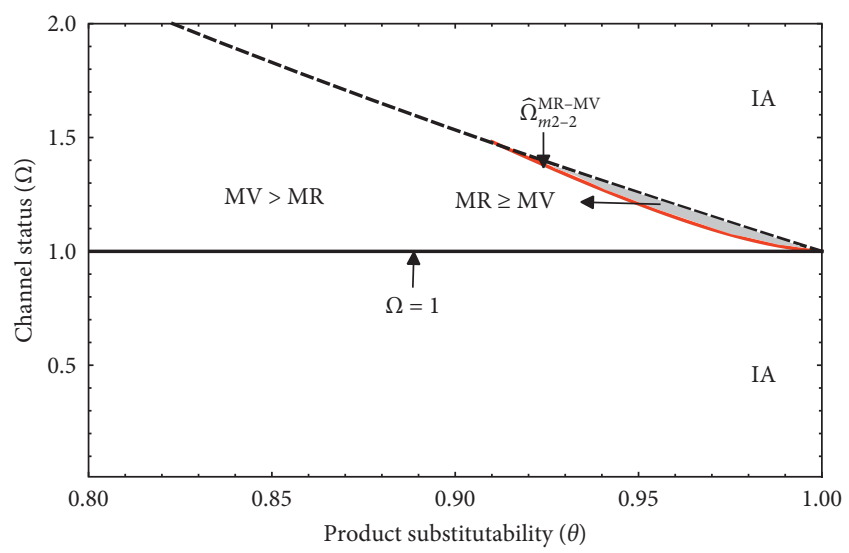

Figure 6: Comparison of manufacturer 2 profits under MS.

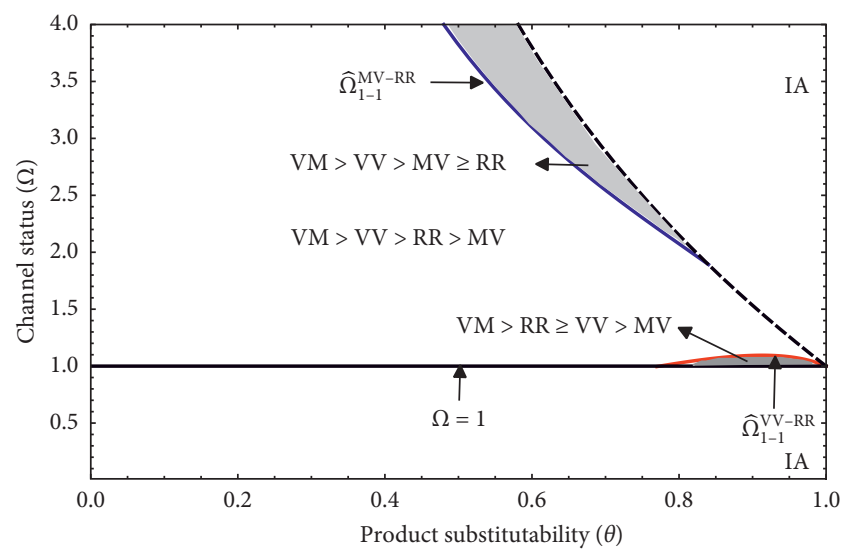

FIGURE 7: Comparison with respect to channel 1 efficiency among all subgames.

differentiated from a vertical competition perspective. Through a representative graph, Figure 8, it can be observed that the channel 2 can be better off than the channel 1 if the channel status is relatively small (e.g., $\pi_{2}^{\mathrm{MM}} \geq \pi_{1}^{\mathrm{MM}}$ if $1<\Omega<\widehat{\Omega}_{1-2}^{\mathrm{MM}-\mathrm{MM}}$ ). Moreover, if the channel status becomes large, the leader is benefitted more than the follower, although he/she sets a higher retail price than the follower's (e.g., $p_{1}^{\mathrm{MM}}>p_{2}^{\mathrm{MM}}$ and
$\pi_{1}^{\mathrm{MM}}>\pi_{2}^{\mathrm{MM}}$ if $\left.\widehat{\Omega}_{1-2}^{\mathrm{MM}-\mathrm{MM}}<\Omega<\Omega_{u}\right)$. In practice, Surface Pro 4 and iPad Pro are similar portable personal computers manufactured by Microsoft and Apple, respectively, and the two competitive electronic products are launched at different times. In particular, Surface Pro 4 sets its selling price later to obtain the second-mover advantage. However, the second-mover advantage only exists for the follower channel when the related channel status is relatively small (note that Xiao et al. [42] show that the first-mover advantage no longer exists if the market is partially covered). Hence, Apple could make more profits than Microsoft due to the larger base market share advantage, although the retail price of iPad Pro is more expensive. This result implies that the timing of product coming into the market and the related channel status have a significant effect on the performance of firms. Another example is the smartphone sector such as HUAWEI Mate series is often launched after Apple to obtain the second-mover advantage. Nevertheless, they should realize that waiting is risky for the firm with a lower base market and profitable only for firm with a higher base market. Therefore, our analysis offers some important decision support on when and how to make pricing policies under different price leadership scenarios.

Similar to Corollary 1, comparing the optimal solutions under symmetric relative channel status, $\Omega=1$, give us an interesting result.

Corollary 2. The follower channel could be always better off if he/she has roughly the same market share as the leader channel (e.g., $\Omega=1$ ).

Corollary 2 indicates that the leader channel will not have an incentive to play the leader's role if the follower channel has the relatively large base market. This result is straightforward. It suggests that a channel into the market later has the late-mover advantage, which is consistent with the previous studies $[19,43]$. In other words, the firm that sets its selling price in a later move earns a higher profit than the firm that sets its price in an earlier move. It is possible that no one wants to move first in a game of perfect information, which would result in a lower profit level as decisions are exposed to the competitors. Furthermore, it is worth noting that the firstmover advantage has been discussed in Choi and Fredj [44], they demonstrate that there exists a first-mover advantage for the retailer when the market is fully covered.

\section{Extensions}

Besides examining the outcomes for the individual firms in the competing supply chains, we also investigate overall supply chain performance and outcomes for the end consumer in the following subsections.

6.1. Social Welfare. We define the entire supply chain efficiency as the Social welfare. Given the symmetric setting for the channel status, we can easily find that $\mathrm{NMR}=\mathrm{NRM}=\mathrm{NRR}, \mathrm{NMV}=\mathrm{NVM}=\mathrm{NVR}=\mathrm{NRV}, \mathrm{MM}=$ $\mathrm{RR}=\mathrm{MR}=\mathrm{RM}, \mathrm{VM}=\mathrm{VR}$, and $\mathrm{MV}=\mathrm{RV}$. Hence, comparing the profits among NMM, NRR, NMV, NVV, MM, VM, 


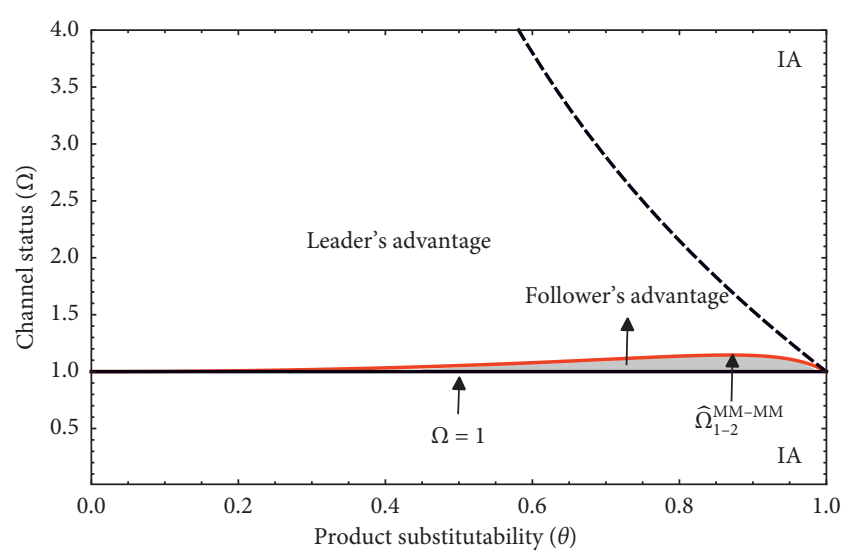

Figure 8: Comparison between channel 1 and channel 2 profits under MM.

$\mathrm{MV}$, and VV with contour plots gives us the following theorem.

Theorem 4. The whole supply chain performs better under Scenario NVV/VV if product substitutability is sufficiently low. However, as product substitutability grows, it can be worse off.

Theorem 4 reveals that Scenario NVV is the dominant strategy for the entire supply chain when product substitutability is lower. Nevertheless, Scenario NVV could make the supply chain get the worst performance, especially under higher substitutability. The rationale is explained as follows. When product substitutability is lower, each member behaves more like a monopolist, and thus the entire supply chain can benefit from $\mathrm{VN}$ game. As the product substitutability grows, the competition between channels becomes more drastic, each member always pursues for taking the role of leader to avoid the loss of own profit, but the Nash member's loss by becoming the follower is greater than the leader's gain, and hence, the entire supply chain can be worse off. This result is consistent with most of the previous studies (Choi [41]). Interestingly, when product substitutability is relatively high such that $\theta \in[0.76,1)$, Scenario MM could be the best for the entire supply chain. We graphically presented this insight in Figure 9. The practical implication for policy makers is that they should understand that Scenario MM could be more beneficial for the society when product substitutability is high. Furthermore, the differences of the entire supply chain efficiency under different price leadership scenarios become smaller as the product substitutability grows.

6.2. Consumer Welfare. We use $U$ to denote Consumer welfare, which superscripted with the different price leadership scenarios being used, and the consumer welfare is based on the utility of the representative consumer. For simplicity, we only compare these optimal solutions among different price leadership scenarios under symmetric relative channel status. We can easily obtain the results that $U^{\mathrm{NRR}}=U^{\mathrm{NMR}}=U^{\mathrm{NRM}}, \quad U^{\mathrm{NVM}}=U^{\mathrm{NVR}}=U^{\mathrm{NMV}}=U^{\mathrm{NRV}}$, $U^{\mathrm{MM}}=U^{\mathrm{MR}}=U^{\mathrm{RM}}=U^{\mathrm{RR}}, U^{\mathrm{VM}}=U^{\mathrm{VR}}$, and $U^{\mathrm{VM}}=U^{\mathrm{VR}}$. Similar to Theorem 4 , we have the following result.

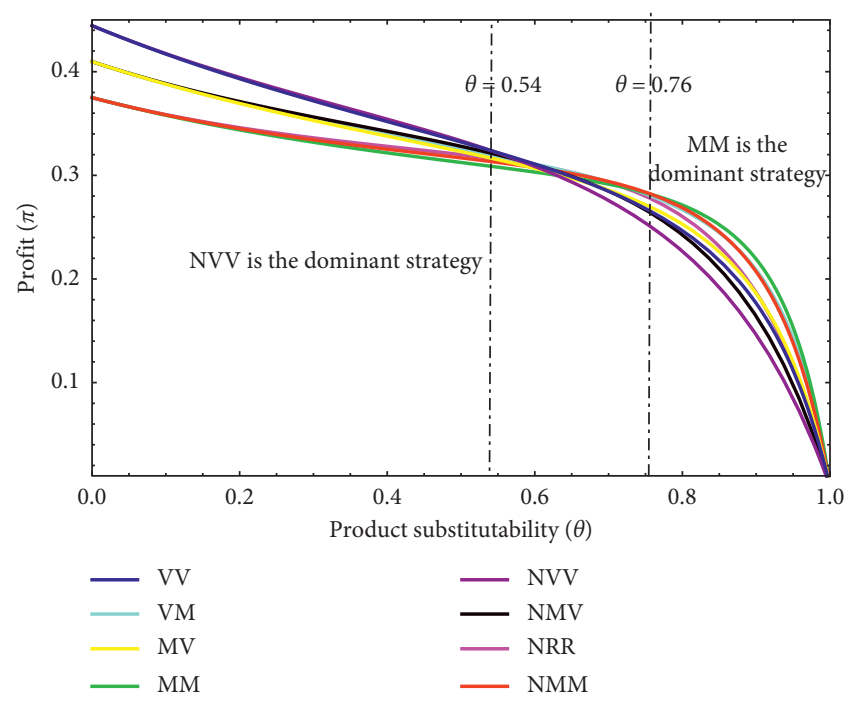

FIgURE 9: Comparison with respect to the entire of supply chain efficiency among all subgames.

Theorem 5. Consumers prefer Scenario NVV the most, while prefer Scenario MM least regardless of product substitutability.

Theorem 5 is straightforward. It suggests that consumers obtain more utility when the two channels are absence of leaders, especial under Case 1 (e.g., NVV), while get worse utility when both channels are denominated by either a manufacturers or a retailer, especial under Case 2 (e.g., MM, MR, RM, and RR). As depicted in Figure 10, the differences of consumer utility under different scenario become larger as the product substitutability grows. Interestingly, the differences become smaller when the product substitutability is relatively high. Further, consumer utility increases in the product substitutability, which implies that higher product substitutability could result in more utility for consumers regardless of the price leadership scenarios. Additionally, Scenario NVM could make consumers get more utility than that under Scenario VV if the product substitutability is relatively high (e.g., $\theta \in[0.76,1))$. In general, Case 1 as a whole is more beneficial to consumers than Case 2 .

\section{Conclusion and Discussion}

This paper investigates the effects of product substitutability and relative channel status on pricing competition under different price leadership scenarios at both vertical and horizontal levels. We consider the symmetric price leadership scenario as well as the asymmetric price leadership scenario. Unlike the previous study of asymmetric price leadership scenario in monopoly common retailer channel $[3,9]$, we examine a dual exclusive channel system with and without horizontal Stackelberg competition, where each manufacturer distributes its goods through a single exclusive retailer but two goods are substitute. Moreover, we employ a linear demand based on the utility function of a representative consumer, which has been widely utilized in economics, marketing, and other related literatures [33, 45, 46]. Our analysis is based on game theory models and reveals 


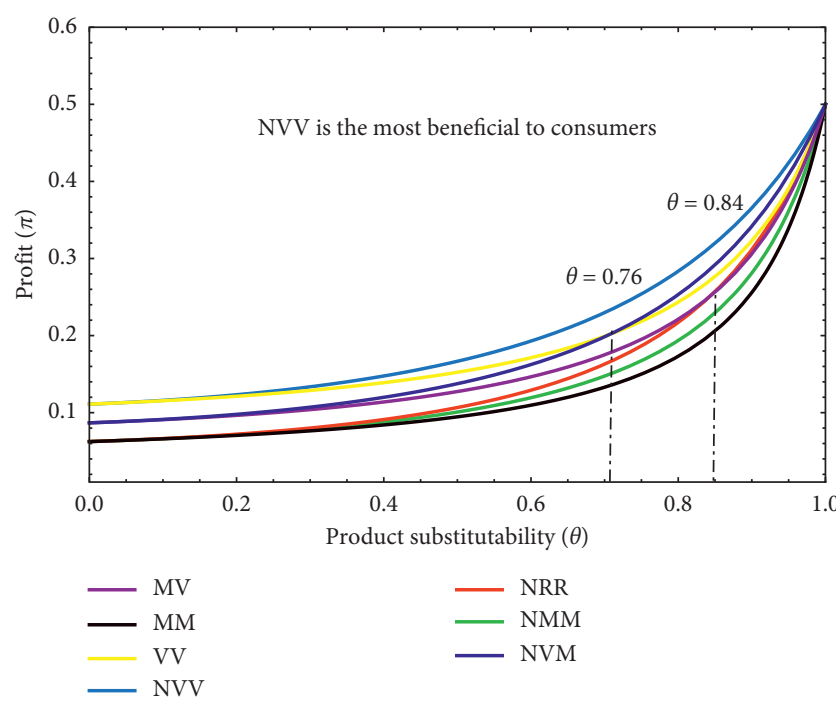

Figure 10: Comparison with respect to consumer welfare among all subgames.

several interesting observations and implications by comparing the different subgames.

From the channel member perspective, under Case 1, the symmetric leadership is always the dominant strategy for either two manufacturers or two retailers regardless of the asymmetric related channel status and the product substitutability. If the asymmetric price leadership situation is considered, it will give us interesting results that are new to the literature, being a Stackelberg leader results in higher profit as well as the equilibrium for either two manufacturers or two retailers from the horizontal competition perspective. Nevertheless, the equilibrium becomes the vertical Nash game from the vertical competition perspective. Further, the retailer 2 with the larger base market can be better off even if dominated by the manufacturer. In other words, the retailer could minimize its disadvantage of being a follower by larger base market advantage and higher product substitutability. By contrast, under Case 2, the symmetric leadership is not always the dominant strategy for either two manufacturers or two retailers.

In general, both two manufacturers and two retailers always archive higher profit by playing the Stackelberg leader, especially under the symmetric leadership situation. From the whole channel perspective, a channel as a whole is profitable in the absence of a price leader. To differ from previous works for a single channel and a monopoly common retailer channel, our analysis demonstrates that whether the leadership is profitable for the whole channel depends on the level of the product substitutability as well as the asymmetric related channel status. For example, under Case 1, given a symmetric status setting, the channels are worse off in the presence of leadership if the product substitutability is sufficiently low. However, as the product substitutability increases, they can be better off from the leadership. If we remove the symmetric status setting, the

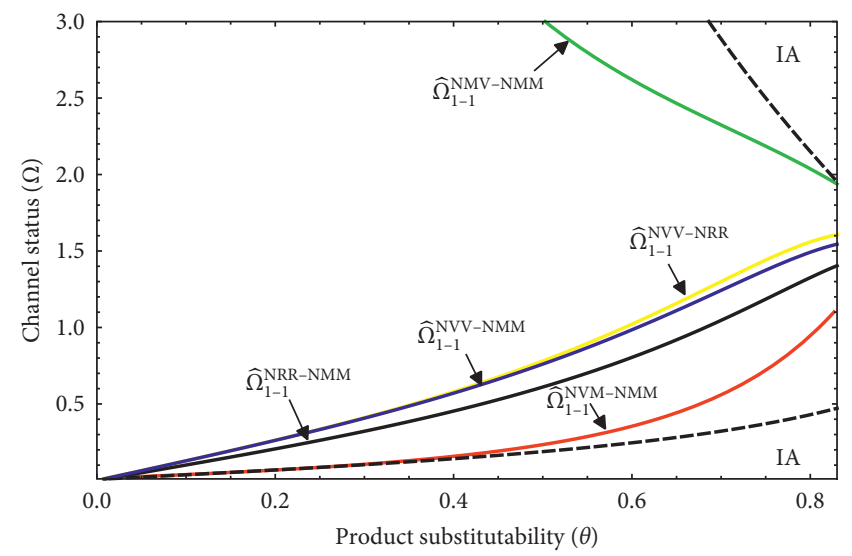

FIGURE 11: Comparison with respect to channel 1 efficiency among all Subgames.

efficiency of the channels is mainly related to the level of the asymmetric related channel status, while little related to the competition degree of product sustainability. Thus, the channel could limit the disadvantage by the higher product substitutability or the lower asymmetric related channel status. Under Case 2, the leader channel has no incentive to play the Stackelberg leader if he has roughly the same market share as the follower channel. This is because the channel into the market later has the late-mover advantage, this explains why rivals prefer not to reveal decisions on prices, bid rates, and contracts, as this information represents bargaining power. Likewise, if the leader channel has the relative larger market share, he always has an incentive to be the leader. This result means that whether the late-mover advantage and the first-mover advantage exist depends on the relative channel status. From the whole supply chain perspective, it can be worse off under Nash game if product substitutability is relative high. Finally, the leadership has a negative impact on the utility of consumers, especially under Case 2.

Our study has practical implications that can be used as decision support. The manager must pay close attention to all firms' supply chain relations as a whole. First, they should take into account their own price leadership situation and the competitor's because the performance is affected by its own price leadership situation as well as the competing firm. Second, since the second-mover advantage depends on the level of the relative channel status. Hence, they should realize that waiting is risky for the firm with a lower base market and is profitable only for firm with a higher base market. The most notable implication for firms is that they should take into account not only the price leadership situation but also the related channel status. This is because they can minimize its disadvantage from being a follower by the larger base market advantage. Furthermore, the policy-makers should understand that the vertical Nash game is not always beneficial to the society. Moreover, they should encourage the leadership competition if the channel has lower base market, especially the one absence of the leader under Case 1. 
This paper compares the profits of manufacturers, retailers and the entire supply chains at both the vertical and horizontal levels. Our analysis might have some limitations. Firstly, demand has been certain in our model. Although this assumption is consistent with the related literature for tractability, it would be intriguing to investigate how demand uncertainty affects the channel member's decision. Previous studies have shown that different demand functions could result in opposite implications for some important issues such as channel leadership and product line pricing $[4,41]$. Secondly, we ignored some other significant factors, such as the operation and production costs. Finally, this paper can be extended in several directions. For instance, further studies could consider a duopoly common retailers channel model with different demand functions, although the computational complexity could be increased significantly.

\section{Appendix}

Appendix A is the derivation of equilibrium solutions for the asymmetric price leadership scenarios and includes all proofs for the main findings of the paper.

\section{A.}

A.1. Since the derivation of equilibrium solutions under Case 1 is similar to the previous literature, such as Choi [1] and Chung and Lee's study [3]. Thus, the derivation for these scenarios is straightforward. Here, we only present the derivation for the asymmetric price leadership scenarios under Case 2.

Scenario MR. In Scenario MR, M1 announces the wholesale price in the first stage, $\mathrm{R} 1$ decides the retail price in the second stage, $\mathrm{R} 2$ decides the retail price in the third stage, and M2 determines the wholesale price in the fourth stage. It is worth noting that M2 conditions his wholesale price on the retail margin, and

$$
w_{2}\left(m_{2}, p_{1}\right)=\frac{1}{2}\left(A_{2}-\theta A_{1}-m_{2}+\theta p_{1}\right) .
$$
and

$\mathrm{R} 2$ Uses the reaction function of M2 to set its retail price,

$$
p_{2}\left(p_{1}\right)=-\frac{3}{4}\left(\theta A_{1}-A_{2}-\theta p_{1}\right)
$$

R1 sets a retail price condition on the wholesale price of $\mathrm{M} 1$ as well as the response function of R2. Similarly, we have R1's best response function:

$$
p_{1}\left(w_{1}\right)=\frac{\left(4-3 \theta^{2}\right) A_{1}-\theta A_{2}+\left(4-3 \theta^{2}\right) w_{1}}{2\left(4-3 \theta^{2}\right)} .
$$

The Hessian matrix of the retailers' profit maximization problem is negative definite. From Eq. (A.2) and Eq. (A.3), we obtain $\mathrm{M1}^{\prime}$ optimal wholesale price as follows:

$$
w_{1}^{\mathrm{MR}^{*}}=\frac{\left(4-3 \theta^{2}\right) A_{1}-\theta A_{2}}{2\left(4-3 \theta^{2}\right)} .
$$

According to the above analysis, it is easy to obtain the other equilibrium solutions.

Certainly, from the nonnegative demand constraints,

$$
\left(4-3 \theta^{2}\right) A_{1} \geq \theta A_{2} \text {. }
$$

Scenario MV. In Scenario MV, M1 sets the wholesale price in the first stage, $\mathrm{R} 1$ determines the retail price in the second stage, M2 and R2 simultaneously make their pricing decision in the third stage. It is easy to get M2's and R2's reaction functions:

$$
w_{2}\left(p_{1}\right)=-\frac{1}{3}\left(\theta A_{1}-A_{2}-\theta p_{1}\right), m_{2}\left(p_{1}\right)=-\frac{1}{3}\left(\theta A_{1}-A_{2}-\theta p_{1}\right) .
$$

R1 decides its retail price using the response function of $\mathrm{R} 2$, conditional on the wholesale price of $\mathrm{M} 1, \mathrm{R} 1$ 's reaction function is derived as

$$
p_{1}\left(w_{1}\right)=\frac{\left(3-2 \theta^{2}\right) A_{1}-\theta A_{2}+\left(3-2 \theta^{2}\right) w_{1}}{6-4 \theta^{2}} \text {. }
$$

Similar to Scenario MR, M1's optimal wholesale price is derived as

$$
w_{1}^{\mathrm{MV}^{*}}=\frac{\left(3-2 \theta^{2}\right) A_{1}-\theta A_{2}}{6-4 \theta^{2}}
$$

Furthermore, the nonnegative demand requires

$$
\left(3-2 \theta^{2}\right) A_{1} \geq \theta A_{2} \text {. }
$$

Scenario $R V$. In Scenario $\mathrm{RV}, \mathrm{R} 1$ chooses a retail price using M1's response function in the first stage. M1 decides its wholesale price using R2's response function in the second stage. M2 and R2 simultaneously determine their optimal prices in the third stage. Similarly, M2's and R2's reaction functions are derived as

$$
\begin{aligned}
& w_{2}\left(p_{1}\right)=-\frac{1}{3}\left(\theta A_{1}-A_{2}-\theta p_{1}\right), \\
& m_{2}\left(p_{1}\right)=-\frac{1}{3}\left(\theta A_{1}-A_{2}-\theta p_{1}\right) .
\end{aligned}
$$

M1 sets its wholesale price conditional on the retail margin of R1 and M1's reaction function can be derived:

$$
w_{1}\left(m_{1}\right)=\frac{\left(3-2 \theta^{2}\right) A_{1}-\theta A_{2}-\left(3-2 \theta^{2}\right) m_{1}}{6-4 \theta^{2}} \text {. }
$$

Similarly, R1's optimal retail price is derived as

$$
p_{1}^{\mathrm{RV}^{*}}=\frac{\left(9-6 \theta^{2}\right) A_{1}-3 \theta A_{2}}{12-8 \theta^{2}}
$$

Moreover, the nonnegative demand requires 


$$
\left(3-2 \theta^{2}\right) A_{1} \geq \theta A_{2}
$$

A.2. This section explores additional properties for the wholesale price and retail price and demand in Case 1 (to save space, we only provide the analysis in Case 1). Comparing the wholesale prices and retail prices among different price leadership scenarios under symmetric relative channel status gives us the following lemma.

Lemma 4. Given a price leadership situation, the wholesale price 1 and retail price 1 have the same rank orders, that is,

(i) Under MS, the rank order is $\varphi_{1}^{N M M}>\varphi_{1}^{N M R}>\varphi_{1}^{N M V}$ regardless of $P S$

(ii) Under $R S$, the rank order is $\varphi_{1}^{N R R}=\varphi_{1}^{N R M}>\varphi_{1}^{N R V}$ regardless of $P S$

(iii) Under VN, the rank order is $\varphi_{1}^{N V M}=\varphi_{1}^{N V R}>\varphi_{1}^{N V V}$ regardless of $P S$

Here, $\varphi \in\{w, p\}$.

We first compare wholesale 1 and retail price 1 under MS game, the wholesale price (retail price) is the highest in scenario NMM followed by NMR and then NMV. Under RS game, the wholesale price (retail price) follows the same properties that the $w_{1}^{\mathrm{NRR}}=w_{1}^{\mathrm{NRM}}>w_{1}^{\mathrm{NRV}}\left(p_{1}^{\mathrm{NRR}}=p_{1}^{\mathrm{NRM}}>p_{1}^{\mathrm{NRV}}\right)$. The result is intuitive that the manufacturer' pricing power enables him/ her to raise the wholesale price, and the retailer have no choice but to raise the retail price. Interestingly, we find that the wholesale price (retail price) is smallest if the owner channel is dominated by a leader but the competing channel is absence of a leader.

In contrast, we find the similar result for wholesale price 2 (retail price) under MS game, which holds the same rank order (e.g., $\varphi_{2}^{\mathrm{NMM}}>\varphi_{2}^{\mathrm{NMR}}>\varphi_{2}^{\mathrm{NMV}}$ and $\varphi \in\{w, p\}$ ). But for wholesale price 2 under RS or VN game, many of these results are reverse, the rank orders is $w_{2}^{\mathrm{NRM}}>w_{2}^{\mathrm{NRV}}>w_{2}^{\mathrm{NRR}}$ or $w_{2}^{\mathrm{NVM}}>w_{2}^{\mathrm{NVV}}>w_{2}^{\mathrm{NVR}}$. This result is counter-intuitive, for instance, the wholesale price 2 is the largest under Scenario NRM, while the retail price 2 under Scenario NRM is the same as that under NRR. More interestingly, we find that the prices hold the same rank orders for the two channels, that is, $p_{i}^{\mathrm{NMM}}>p_{i}^{\mathrm{NMR}}>p_{i}^{\mathrm{NMV}}, \quad p_{\mathrm{i}}^{\mathrm{NRR}}=P_{i}^{\mathrm{NRM}}>p_{i}^{\mathrm{NRV}}, \quad$ and $p_{i}^{\mathrm{NVR}}=P_{i}^{\mathrm{NVM}}>p_{i}^{\mathrm{NVV}}, i \in\{1,2\}$. But the price gap becomes larger if we switch indices 1 and 2 of the equilibrium solutions (e.g., $p_{2}^{\mathrm{NRR}}-P_{2}^{\mathrm{NRV}}>p_{1}^{\mathrm{NRR}}-P_{1}^{\mathrm{NRV}}$ ).

Comparing the wholesale prices and retail prices under all of scenarios and combining with Lemma 4 discussed above, the theorem can be obtained as follows.

Theorem 6. The wholesale price $i$ is the lowest if channel $i$ is dominated by the retailer $i$ and the competing channel has absence of a leader (e.g., NRV) and the retail price $i$ is lowest if both channels have absence of leaders (e.g., NVV). However, both the wholesale price $i$ and the retail price $i$ are the highest if both manufacturers dominate the channels (e.g., NMM).
Theorem 6 shows that the wholesale price 1 (2) is lowest in Scenario NRV (NVR), it is intuitive that a Stackelberg leader by a retailer results in higher retail price but lower wholesale price. The retail price 1 (2) is lowest in Scenario NVV, which is consistent with the previous studies (Zhang et al. [8]). However, both the wholesale price 1 (2) and the retail price 1 (2) are the highest in Scenario NMM. Under Scenario NMM, each manufacturer foresees the corresponding retailer's reaction to set a higher wholesale price and the higher wholesale price results in the increasing of the retail price. This result is counter-intuitive, which is contrary to Chung and Lee [3] and Choi's studies [41], who point out that the retail price is generally highest if the retailer is the leader. This is because, in their model, they consider a monopoly common retailer model and there is no competition at the retail level.

In addition, demand is the most important factor in marketing. By comparing the demands among different price leadership scenarios, we have the following.

Lemma 5. Given a price leadership situation, the demand $i$ has the following rank orders, that is,

(i) Under MS, the rank order is $D_{1}^{N M R}>D_{1}^{N M M}>D_{1}^{N M V}$ and $D_{2}^{N M V}>D_{2}^{N M R}>D_{2}^{N M M}$ regardless of $P S$

(ii) Under $R S$, the rank order is $D_{1}^{N R R}=D_{1}^{N R M}>D_{1}^{N R V}$ and $D_{2}^{N R V}>D_{2}^{N R R}=D_{2}^{N R M}$ regardless of $P S$

(iii) Under VN, the rank order is $D_{1}^{N V R}=D_{1}^{N V M}>D_{1}^{N V V}$ and $D_{2}^{N V V}>D_{2}^{N V R}=D_{2}^{N V M}$ regardless of $P S$

Lemma 5 shows that the demands 1 is larger in Scenario NMR, Scenario NRR/NRM, and Scenario NVR/NVM respectively, given a leadership situation for channel 1 . In contrast, these results are different for demand 2: here, we find that the demand 2 is larger in Scenario NMV, Scenario NRV, and Scenario NVV, respectively. These results indicate that the demand 1 (2) is affected by its own channel leadership situation as well as that of the competing channel. Demand is assumed to be decreasing in its price and increasing in the competing channel's price and combined with the equilibrium retail prices discussed above. Associated with the retail prices, demand levels are equal under Scenario NRR/NRM (NVR/NVM) (e.g., $D_{1}^{\text {NRR }}=D_{1}^{\text {NRM }}$ and $\left.D_{1}^{\mathrm{NVR}}=D_{1}^{\mathrm{NVM}}\right)$. Moreover, our analysis also shows that the price gap between two channels in Scenario NRM (NVM) is larger than that in Scenario NRV (NVV) (e.g., $\left.p_{2}^{\mathrm{NRM}}-P_{1}^{\mathrm{NRM}}>p_{2}^{\mathrm{NRV}}-P_{1}^{\mathrm{NRV}}\right)$; hence, Scenario NRM (NVM) always results in larger demand than that in Scenario NRV (NVV).

Similar to Theorem 6, Theorem 7 can be derived.

Theorem 7. Firms have the smallest demand if their own channel is dominated by either a manufacturer or a retailer and the rival channel is absence of a leader (e.g., NMV or $N R V)$, while gain the largest demand if their own channel is dominated by no channel members and the rival channel is dominated by either a manufacturer or a retailer (e.g., NVM or NVR). 
Theorem 7 indicates that the demand 1 is smallest in Scenario NMV/NRV and largest in Scenario NVM/NVR. Likewise, it is easy to find that the demand 2 has the opposite results. This result occurs because being a Stackelberg leader by either the manufacturer or the retailer results in higher price and thereby has a negative effect on the demand. The result is consistent with Chung and Lee [3] who also note that the demand 2 is largest when manufacturer 1 acts as a leader, though we consider a dual exclusive channel and they consider a monopoly common retailer channel. In contrast, Zhang et al. [8] demonstrate that the demand is highest in Scenario NVV and lowest in Scenario NMM. Moreover, the firms as a whole have the largest demand in VN game regardless of the leadership situation of their competitors (e.g., $D_{1}^{\mathrm{NVj}}>D_{1}^{\mathrm{NMj}}$ or $D_{1}^{\mathrm{NVj}}>D_{1}^{\mathrm{NR} / \mathrm{j}}$ ). This observation has been explained by Lee and Staelin [4] that being a price leader has a negative effect on demand in the vertical strategic substitutability environment.

A.3. To simplify our exposition, we organized the discussion of this analysis by comparing the equilibrium results of different power structures for channel 1 while holding fixed the price leadership scenario for channel. We first compare the equilibrium prices and profits among different price leadership scenarios under symmetric relative channel status, that is $\Omega=1$. Then, we examine the similar problems given asymmetric setting, that is $\Omega \neq 1$. Note that we only examine the asymmetric scenario for Case 2, which holds the condition $\Omega \geq 1$.

Proof of Lemma 1. To facilitate our discussion, we temporarily define $\Delta \pi_{m 1}^{\mathrm{NMR}^{*}-\mathrm{NMV}^{*}}(\theta)$ as manufacturer 1's profit in Scenario NMR minus the one in Scenario NMV. Firstly, comparing the equilibrium profits for the manufacturer 1 , given a price leadership situation, yields the following results,
(i) $\pi_{m 1}^{\mathrm{NMM}^{*}}>\pi_{m 1}^{\mathrm{NMR}^{*}}>\pi_{m 1}^{\mathrm{NMV}^{*}}$ for any $\theta \in(0,1)$;
(ii) $\pi_{m 1}^{\mathrm{NRR}^{*}}=\pi_{m 1}^{\mathrm{NRM}^{*}}>\pi_{m 1}^{\mathrm{NRV}^{*}}$ for any $\theta \in(0,1)$;
(iii) $\pi_{m 1}^{\mathrm{NVR}^{*}}=\pi_{m 1}^{\mathrm{NVM}^{*}}>\pi_{m 1}^{\mathrm{NVV}^{*}}$ for any $\theta \in(0,1)$.

Here, we start with MS game, since $\Delta \pi_{m 1}^{\mathrm{NMM}^{*}-\mathrm{NMR}^{*}}(\theta)=$ $\theta^{3}\left(4-6 \theta+\theta^{2}+\theta^{3}\right) /(2-\theta)(1+\theta)(4-3 \theta)^{2}\left(4-\theta-2 \theta^{2}\right)^{2}$ $>0$ and $\Delta \pi_{m 1}^{\mathrm{NMR}^{*}-\mathrm{NMV}^{*}}(\theta)=\theta(1-\theta)\left(24-\theta-12 \theta^{2}\right) / 18$ $(4-3 \theta)^{2}(1+\theta)\left(2-\theta^{2}\right)^{2}>0$, we obtain $\pi_{m 1}^{\mathrm{NMV}^{*}}<\pi_{m 1}^{\mathrm{NMR}^{*}}$ $<\pi_{m 1}^{\mathrm{NMM}^{*}}$. Similarly, under RS and VN games, we have $\pi_{m 1}^{\mathrm{NRR}^{*}}=\pi_{m 1}^{\mathrm{NRM}^{*}}>\pi_{m 1}^{\mathrm{NRV}^{*}}$ and $\pi_{m 1}^{\mathrm{NVR}^{*}}=\pi_{m 1}^{\mathrm{NVM}^{*}}>\pi_{m 1}^{\mathrm{NVV}^{*}}$. This is because $\Delta \pi_{m 1}^{\mathrm{NRR}^{*}-\mathrm{NRV}^{*}}(\theta)=\theta(1-\theta)\left(24-\theta-12 \theta^{2}\right) / 36(4-$ $3 \theta)^{2}(1+\theta)\left(-2+\theta^{2}\right)^{2}>0$ and $\Delta \pi_{m 1}^{\mathrm{NVV}^{*}-\mathrm{NVR}^{*}}(\theta)=-\theta(1$ $-\theta)\left(24+\theta-12 \theta^{2}\right) / 36(3-2 \theta)^{2}(1+\theta)\left(2-\theta^{2}\right)^{2}<0$. Moreover, $\pi_{m 1}^{\mathrm{NVM}^{*}}=\pi_{m 1}^{\mathrm{NVR}^{*}}$ and $\pi_{m 1}^{\mathrm{NVR}^{*}}=\pi_{m 1}^{\mathrm{NVM}^{*}}$.

We then compare the profits for the retailer 1 , and a similar process obtains the following results:
(i) $\pi_{r 1}^{\mathrm{NMR}^{*}}>\pi_{r 1}^{\mathrm{NMM}^{*}}>\pi_{r 1}^{\mathrm{NMV}^{*}}$ for any $\theta \in(0,1)$;
(ii) $\pi_{r 1}^{\mathrm{NRR}^{*}}=\pi_{r 1}^{\mathrm{NRM}^{*}}>\pi_{r 1}^{\mathrm{NRV}^{*}}$ for any $\theta \in(0,1)$;

(iii) $\pi_{r 1}^{\mathrm{NVM}^{*}}=\pi_{r 1}^{\mathrm{NVR}^{*}}>\pi_{r 1}^{\mathrm{NVV}^{*}}$ for any $\theta \in(0,1)$.

Similar methods yield $\Delta \pi_{r 1}^{\mathrm{NMM}^{*}-\mathrm{NMR}^{*}}(\theta)=-\theta^{2}(1-$ $\theta)^{2}\left(16-12 \theta-7 \theta^{2}+5 \theta^{3}\right) /(1+\theta)(4-3 \theta)^{2}(2-\theta)^{2}(4-\theta-$ $\left.2 \theta^{2}\right)^{2}<0 \quad$ and $\Delta \pi_{r 1}^{\mathrm{NMM}^{*}-\mathrm{NMV}^{*}}(\theta)=\theta(1-\theta)(96$ $\left.-148 \theta-84 \theta^{2}+231 \theta^{3}+16 \theta^{4}-120 \theta^{5}+20 \theta^{7}\right) / 36(1+\theta)$ $-\theta)^{2}\left(2-\theta^{2}\right)^{2}\left(-4+\theta+2 \theta^{2}\right)^{2}>0$. Therefore, we obtain $\pi_{r 1}^{\mathrm{NMV}^{*}}<\pi_{r 1}^{\mathrm{NMM}^{*}}<\pi_{r 1}^{\mathrm{NMR}^{*}}$. Also, we have, $\pi_{r 1}^{\mathrm{NRV}^{*}}<\pi_{r 1}^{\mathrm{NRR}^{*}}=\pi_{r 1}^{\mathrm{NRM}^{*}}$ and $\pi_{r 1}^{\mathrm{NVV}^{*}}<\pi_{r 1}^{\mathrm{NVM}^{*}}=\pi_{r 1}^{\mathrm{NVR}^{*}}$.

In the following, without loss of generality, we also explore the similar problem for the channel 1 . We have

(i) $\pi_{1}^{\mathrm{NMR}^{*}}>\pi_{1}^{\mathrm{NMM}^{*}}>\pi_{1}^{\mathrm{NMV}^{*}}$ for $\theta \in(0,0.68)$

(ii) $\pi_{1}^{\mathrm{NRR}^{*}}=\pi_{1}^{\mathrm{NRM}^{*}}>\pi_{1}^{\mathrm{NRV}^{*}}$ for any $\theta \in(0,1)$

(iii) $\pi_{1}^{\mathrm{NVM}^{*}}=\pi_{1}^{\mathrm{NVR}^{*}}>\pi_{1}^{\mathrm{NVV}^{*}}$ for any $\theta \in(0,1)$

The proof of the channel 1 is similar to that of the manufacturer 1 (the retailer 1); thus, we omit the details.

Proof of Theorem 1. To describe the stability of the different price leadership structures, we are positioned to compare the equilibrium profits under all game scenarios. Combining with Lemma 1 , it is easy to have the following results:

$$
\begin{aligned}
& \text { (i) } \pi_{m 1}^{\mathrm{NMM}^{*}}>\pi_{m 1}^{\mathrm{NMR}^{*}}>\pi_{m 1}^{\mathrm{NMR}^{*}}>\pi_{m 1}^{\mathrm{NVM}^{*}}>\pi_{m 1}^{\mathrm{NVV}^{*}}>\pi_{m 1}^{\mathrm{NRR}^{*}}> \\
& \pi_{m 1}^{\mathrm{NRV}} \text { for any } \theta \in(0,1) \\
& \text { (ii) } \pi_{r 1}^{\mathrm{NRR}^{*}}>\pi_{r 1}^{\mathrm{NRV}^{*}}>\pi_{r 1}^{\mathrm{NVM}^{*}}>\pi_{r 1}^{\mathrm{NVV}^{*}}>\pi_{r 1}^{\mathrm{NMR}^{*}}>\pi_{r 1}^{\mathrm{NMM}^{*}}> \\
& \pi_{r 1}^{\mathrm{NMV}^{*}} \text { for any } \theta \in(0,1) \\
& \text { (iii) } \pi_{1}^{\mathrm{NVM}^{*}}>\pi_{1}^{\mathrm{NRR}^{*}} \geq \pi_{1}^{\mathrm{NVV}^{*}}>\pi_{1}^{\mathrm{NMM}^{*}}>\pi_{1}^{\mathrm{NMV}^{*}} \text { for } \theta \in \\
& {[0.59,0.61) \text {, }} \\
& \pi_{1}^{\mathrm{NVM}^{*}}>\pi_{1}^{\mathrm{NRR}^{*}}>\pi_{1}^{\mathrm{NMM}^{*}} \geq \pi_{1}^{\mathrm{NVV}^{*}}>\pi_{1}^{\mathrm{NMV}^{*}} \text { for } \theta \epsilon \\
& {[0.61,0.68) \text {, }} \\
& \pi_{1}^{\mathrm{NVM}^{*}}>\pi_{1}^{\mathrm{NMM}^{*}} \geq \pi_{1}^{\mathrm{NRR}^{*}}>\pi_{1}^{\mathrm{NVV}^{*}}>\pi_{1}^{\mathrm{NMV}^{*}} \text { for } \theta \epsilon \\
& {[0.68,0.82) \text {, }} \\
& \pi_{1}^{\mathrm{NMM}^{*}} \geq \pi_{1}^{\mathrm{NVM}^{*}}>\pi_{1}^{\mathrm{NRR}^{*}}>\pi_{1}^{\mathrm{NVV}^{*}}>\pi_{1}^{\mathrm{NMV}^{*}} \text { for } \theta \in \\
& {[0.82,0.88) \text {, }} \\
& \pi_{1}^{\mathrm{NMM}^{*}}>\pi_{1}^{\mathrm{NRR}^{*}} \geq \pi_{1}^{\mathrm{NVM}^{*}}>\pi_{1}^{\mathrm{NVV}^{*}}>\pi_{1}^{\mathrm{NMV}^{*}} \text { for } \theta \epsilon \\
& {[0.88,0.94) \text {, and }} \\
& \pi_{1}^{\mathrm{NMM}^{*}}>\pi_{1}^{\mathrm{NRR}^{*}}>\pi_{1}^{\mathrm{NVM}^{*}}>\pi_{1}^{\mathrm{NMV}^{*}} \geq \pi_{1}^{\mathrm{NVV}^{*}} \text { for } \theta \in \\
& {[0.94,1)}
\end{aligned}
$$

Here, $\quad \pi_{m 1}^{\mathrm{NVM}^{*}}=\pi_{m 1}^{\mathrm{NVR}^{*},} \quad \pi_{m 1}^{\mathrm{NRR}^{*}}=\pi_{m 1}^{\mathrm{NRM}^{*},}$ $\begin{array}{ll}\pi_{m 1}^{\mathrm{N}}=\pi_{m 1}^{\mathrm{N}}, & \pi_{m 1}^{\mathrm{N} 1}=\pi_{m 1}^{\mathrm{N} 1}, \\ \pi_{r 1}^{\mathrm{NRR}^{*}}=\pi_{r 1}^{\mathrm{NRV}^{*}} \pi_{r 1}^{\mathrm{NVM}^{*}=\pi_{r 1}^{\mathrm{NVR}^{*}},} & \pi_{1}^{\mathrm{NVR}^{*}}=\pi_{1}^{\mathrm{NVM}^{*}}, \\ \pi_{1}^{\mathrm{NMR}^{*}}=\pi_{1}^{\mathrm{NRR}^{*}}=\pi_{1}^{\mathrm{NRM}^{*}}, \text { and } \pi_{1}^{\mathrm{NMV}^{*}}=\pi_{1}^{\mathrm{NRV}^{*}} .\end{array}$

Similar to the proof in Lemma 1, we first compare the equilibrium profits for the manufacturer 1 . Then, we have, $\Delta \pi_{m 1}^{\mathrm{NMV}^{*}-\mathrm{NVM}^{*}}(\theta)=(1-\theta) / 36(1+\theta)\left(2-\theta^{2}\right)>0$ and

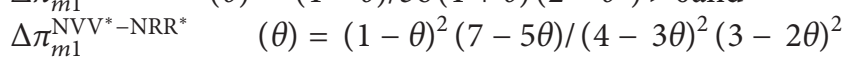
$(1+\theta)>0$. Combining with the results of Lemma 1 , it is easy to find that $\pi_{m 1}^{\mathrm{NMM}^{*}}>\pi_{m 1}^{\mathrm{NMR}^{*}}>\pi_{m 1}^{\mathrm{NMR}^{*}}>\pi_{m 1}^{\mathrm{NVM}^{*}}>$ $\pi_{m 1}^{\mathrm{NVV}^{*}}>\pi_{m 1}^{\mathrm{NRR}^{*}}>\pi_{m 1}^{\mathrm{NRV}^{*}}$.

We then compare these profits for the retailer 1, Similarly, we obtain $\Delta \pi_{r 1}^{\mathrm{NRV}^{*}-\mathrm{NMR}^{*}}(\theta)=(1-\theta)\left(72-24 \theta-71 \theta^{2}\right.$ $\left.+12 \theta^{3}+18 \theta^{4}\right) / 18(4-3 \theta)^{2}(1+\theta)\left(2-\theta^{2}\right)^{2}>0, \Delta \pi_{r 1}^{\mathrm{NRV}^{*}-\mathrm{NVM}^{*}}$ $(\theta)=1-\theta / 36(1+\theta)\left(2-\theta^{2}\right)>0, \quad$ and $\Delta \pi_{r 1}^{\mathrm{NVV}^{*}-\mathrm{NMR}^{*}}$ $(\theta)=(7-5 \theta) \quad(1-\theta)^{2} /(4-3 \theta)^{2}(3-2 \theta)^{2} \quad(1+\theta)>0$. Thus, the above relations show that $\pi_{r 1}^{\mathrm{NRR}^{*}}$ $>\pi_{r 1}^{\mathrm{NRV}^{*}}>\pi_{r 1}^{\mathrm{NVM}^{*}}>\pi_{r 1}^{\mathrm{NVV}^{*}}>\pi_{r 1}^{\mathrm{NMR}^{*}}>\pi_{r 1}^{\mathrm{NMM}^{*}}>\pi_{r 1}^{\mathrm{NMV}^{*}}$. 
Furthermore, we can obtain the results for the channel 1 as follows: $\Delta \pi_{1}^{\mathrm{NRR}^{*}-\mathrm{NVV}^{*}}(\theta)=-(1-\theta)\left(5-12 \theta+6 \theta^{2}\right)$ / $(4-3 \theta)^{2}(3-2 \theta)^{2}(1+\theta) \leq 0$ if $\theta \in(0,0.59], \Delta \pi_{1}^{\mathrm{NMM}^{*}-\mathrm{NVV}^{*}}$ $(\theta)=-(1-\theta)\left(5-12 \theta+6 \theta^{2}\right) /(4-3 \theta)^{2}(3-2 \theta)^{2}(1+\theta) \leq$ 0 if $\theta \in(0,0.61], \Delta \pi_{1}^{\mathrm{NMM}^{*}-\mathrm{NRR}^{*}}(\theta)=-\theta^{2}\left(16-52 \theta+49 \theta^{2}-\right.$ $\left.\theta^{3}-18 \theta^{4}+6 \theta^{5}\right) /(4-3 \theta)^{2}(2-\theta)^{2}(1+\theta)\left(4-\theta-2 \theta^{2}\right)^{2} \leq 0$ if $\theta \in(0,0.68], \Delta \pi_{1}^{\mathrm{NMM}^{*}-\mathrm{NVR}^{*}}(\theta)=-(1-\theta)\left(160-336 \theta^{2}-\right.$ $\left.64 \theta^{3}+204 \theta^{4}+60 \theta^{5}-35 \theta^{6}-12 \theta^{7}\right) / 18(2-\theta)^{2}(1+\theta)(2-$ $\left.\theta^{2}\right)^{2}\left(4-\theta-2 \theta^{2}\right)^{2} \leq 0$ if $\theta \in(0,0.82], \Delta \pi_{1}^{\mathrm{NRR}^{*}-\mathrm{NVR}^{*}}=-$ $(1+\theta)\left(40-72 \theta^{2}+27 \theta^{4}\right) / 18(4-3 \theta)^{2}(1+\theta)\left(2-\theta^{2}\right)^{2} \leq 0$ if $\theta \in(0,0.88]$, and $\Delta \pi_{1}^{\mathrm{NVV}^{*}-\mathrm{NMV}^{*}}=(1-\theta)\left(15-24 \theta^{2}+\right.$ $\left.8 \theta^{4}\right) / 12(3-2 \theta)^{2}(1+\theta)\left(2-\theta^{2}\right)^{2} \leq 0$ if $\theta \in[0.94,1)$. From the above analysis, we obtain the result of Part (iii).

Proof of Lemma 2. Without loss of generality, we compare the equilibrium profits under MS, RS, and VN games with asymmetric related channel status. Note that the discussion is conditional on the nonnegative demand. From the nonnegative demand constraints, we can get the Common Feasible Area is $\Omega_{1}=\theta / 3-2 \theta^{2}$ and $\Omega_{u}=3-2 \theta^{2} / \theta$, where $\Omega_{1}$ and $\Omega_{u}$ represent the common lower and upper bounds, respectively. Note that the two constraint lines cross when $\theta=1$. Due to the complexity of all boundary values, we use $\widehat{\Omega}$ to denote the boundary values with various superscript and subscript combinations, and throughout this study, we assume $\widehat{\Omega}_{s-1}>\widehat{\Omega}_{s-2}$ (e.g., $\left.\widehat{\Omega}_{m 1-1}^{\mathrm{NMR}-\mathrm{NMV}}>\widehat{\Omega}_{m 1-2}^{\mathrm{NMR}-\mathrm{NMV}}\right)$. Furthermore, to facilitate our discussion, we use PS and AS to denote the product substitutability and asymmetric related channel status, respectively. Similar to that of symmetric scenario, we first compare the profits for manufacturer 1. Given a price leadership situation, we have,
(i) $\pi_{m 1}^{\mathrm{NMM}^{*}}>\pi_{m 1}^{\mathrm{NMR}^{*}}>\pi_{m 1}^{\mathrm{NMV}^{*}}$ regardless of PS and AS
(ii) $\pi_{m 1}^{\mathrm{NRR}^{*}}=\pi_{m 1}^{\mathrm{NRM}^{*}}>\pi_{m 1}^{\mathrm{NRV}^{*}}$ regardless of PS and AS
(iii) $\pi_{m 1}^{\mathrm{NVR}^{*}}=\pi_{m 1}^{\mathrm{NVM}^{*}}>\pi_{m 1}^{\mathrm{NVV}^{*}}$ regardless of PS and AS

Under MS game, to compare Scenarios NMR and NMV, we define $\Delta \pi_{m 1}^{\mathrm{NMR}^{*}-\mathrm{NMV}^{*}}(\Omega, \theta)=\pi_{m 1}^{\mathrm{NMR}^{*}}-\pi_{m 1}^{\mathrm{NMV}^{*}}=0$, and solving $\Delta \pi_{m 1}^{\mathrm{NMR}^{*}-\mathrm{NMV}^{*}}(\Omega, \theta)=0$ yields two roots, that is $\widehat{\Omega}_{m 1-1}^{\mathrm{NMR}-\mathrm{NMV}}=4-3 \theta^{2} / \theta$ and $\widehat{\Omega}_{m 1-2}^{\mathrm{NMR}-\mathrm{NMV}}=28 \theta-15 \theta^{3} / 96-$ $119 \theta^{2}+36 \theta^{4}$. Contour plots clearly demonstrate that $\widehat{\Omega}_{m 1-2}^{\mathrm{NMR}-\mathrm{NMV}}<\Omega_{1}<\Omega_{u}<\widehat{\Omega}_{m 1-1}^{\mathrm{NMR}-\mathrm{NMV}}$ and $\partial^{2} \Delta \pi_{m 1}^{\mathrm{NMR}^{*}-\mathrm{NMV}^{*}}$ $/ \partial \Omega^{2}<0$; hence, Scenario NMR results in higher profits for the manufacturer 1 , that is $\pi_{m 1}^{\mathrm{NMR}^{*}}>\pi_{m 1}^{\mathrm{NMV}^{*}}$. Similarly, we obtain $\pi_{m 1}^{\mathrm{NMM}^{*}}>\pi_{m 1}^{\mathrm{NMR}^{*}}$, due to $\widehat{\Omega}_{m 1-2}^{\mathrm{NMM}-\mathrm{NMR}}$

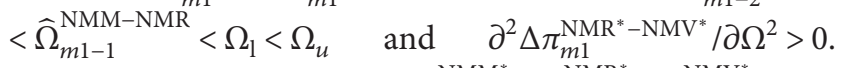
Therefore, we can infer that $\pi_{m 1}^{\mathrm{NMM}^{*}}>\pi_{m 1}^{\mathrm{NMR}^{*}}>\pi_{m 1}^{\mathrm{NMV}^{*}}$ from the above inequality.

Under RS game, we can easily obtain $\pi_{m 1}^{\mathrm{NRR}^{*}}=\pi_{m 1}^{\mathrm{NRM}^{*}}$ from Tables 2 and 3. Solving $\Delta \pi_{m 1}^{\mathrm{NRR}^{*}-\mathrm{NRV}^{*}}(\Omega, \theta)=0$ yields two roots, that is, $\widehat{\Omega}_{m 1-1}^{\mathrm{NRR}-\mathrm{NRV}}=4-3 \theta^{2} / \theta$ and $\widehat{\Omega}_{m 1-2}^{\mathrm{NRR}-\mathrm{NRV}}=28 \theta-15 \theta^{3} / 96-119 \theta^{2}+36 \theta^{4}$. Note that $\widehat{\Omega}_{m 1-2}^{\mathrm{NMM}-\mathrm{NMR}}<\Omega_{1}<\Omega_{u}<\widehat{\Omega}_{m 1-1}^{\mathrm{NMM}-\mathrm{NMR}}$. Therefore, $\pi_{m 1}^{\mathrm{NRR}^{*}}=$ $\pi_{m 1}^{\mathrm{NRM}^{*}}>\pi_{m 1}^{\mathrm{NRV}^{*}}$. Under VN game, using the similar method, we have $\pi_{m 1}^{\mathrm{NVV}^{*}}<\pi_{m 1}^{\mathrm{NVR}^{*}}=\pi_{m 1}^{\mathrm{NVM}^{*}}$.
Next, a similar process obtains the following results for retailer 1: (i) $\pi_{r 1}^{\mathrm{NMR}^{*}} \geq \pi_{r 1}^{\mathrm{NMM}^{*}} \geq \pi_{r 1}^{\mathrm{NMV}^{*}} \quad$ if $\quad \widehat{\Omega}_{r 1-1}^{\mathrm{NMM-NMR}} \leq \Omega \leq$
$\widehat{\Omega}_{r 1-1}^{\mathrm{NMM}-\mathrm{NMV}}$

(ii) $\pi_{r 1}^{\mathrm{NRR}^{*}}=\pi_{r 1}^{\mathrm{NRM}^{*}}>\pi_{r 1}^{\mathrm{NRV}^{*}}$ regardless of PS and AS

(iii) $\pi_{r 1}^{\mathrm{NVR}^{*}}=\pi_{r 1}^{\mathrm{NVM}^{*}}>\pi_{r 1}^{\mathrm{NVV}^{*}}$ regardless of PS and AS

Here, $\widehat{\Omega}_{r 1-1}^{\mathrm{NMM-NMR}}=\theta\left(16-19 \theta^{2}+5 \theta^{4}\right) / 32-58 \theta^{2}+34 \theta^{4}$ $-6 \theta^{6}$ and $\widehat{\Omega}_{r 1-1}^{\mathrm{NMM}-\mathrm{NMV}}=\left(16-12 \theta^{2}-3 \theta^{4}+2 \theta^{6}\right) / 28 \theta-$ $45 \theta^{3}+24 \theta^{5}-4 \theta^{7}$.

We first consider MS game, since $\widehat{\Omega}_{r 1-2}^{\mathrm{NMM-NMR}}<\Omega_{1}<\widehat{\Omega}_{r 1-1}^{\mathrm{NMM-NMR}}<\Omega_{u}$, we have $\pi_{r 1}^{\mathrm{NMM}^{*}}>\pi_{r 1}^{\mathrm{NMR}^{*}}$ if $\Omega_{1}<\Omega<\widehat{\Omega}_{r 1-1}^{\mathrm{NMM} \text { NMR }}$. A similar process obtains $\pi_{r 1}^{\mathrm{NMM}^{*}}$ $>\pi_{r 1}^{\mathrm{NMV}^{*}}$ if $\Omega_{1}<\Omega<\widehat{\Omega}_{r 1-1}^{\mathrm{NMM}-\mathrm{NMV}}$. Next, under RS game, based on the results from Tables 2 and 3, we obtain $\pi_{r 1}^{\mathrm{NRR}^{*}}=\pi_{r 1}^{\mathrm{NRM}^{*}} . \quad$ Meantime, $\quad \widehat{\Omega}_{r 1-2}^{\mathrm{NRR}-\mathrm{NRV}}<\Omega_{1}<\Omega_{u}<$ $\widehat{\Omega}_{r 1-1}^{\mathrm{NRR}-\mathrm{NRV}}$, where $\widehat{\Omega}_{r 1-2}^{\mathrm{NRR}-\mathrm{NRV}}=28 \theta-15 \theta^{3} / 96-119 \theta^{2}+36 \theta^{4}$ and $\widehat{\Omega}_{r 1-1}^{\mathrm{NRR}-\mathrm{NRV}}=4-3 \theta^{2} / \theta$. Thus, $\pi_{r 1}^{\mathrm{NRR}^{*}}=\pi_{r 1}^{\mathrm{NRM}^{*}}>\pi_{r 1}^{\mathrm{NRV}^{*}}$. Lastly, under VN game, because $\widehat{\Omega}_{r 1-2}^{\mathrm{NRR}-\mathrm{NRV}}<\Omega_{\mathrm{l}}$ $<\Omega_{u}<\widehat{\Omega}_{r 1-1}^{\mathrm{NRR}-\mathrm{NRV}}$, where $\widehat{\Omega}_{r 1-2}^{\mathrm{NRR}-\mathrm{NRV}}=3-2 \theta^{2} / \theta, \widehat{\Omega}_{r 1-1}^{\mathrm{NRR}-\mathrm{NRV}}$ $=21 \theta-10 \theta^{3} / 72-85 \theta^{2}+24 \theta^{4}$. Therefore, it can be verified that $\pi_{r 1}^{\mathrm{NVR}^{*}}=\pi_{r 1}^{\mathrm{NVM}^{*}}>\pi_{r 1}^{\mathrm{NVV}^{*}}$.

With similar approach shown for the comparison for channel 1, we have

(i) $\pi_{1}^{\mathrm{NMM}^{*}}>\pi_{1}^{\mathrm{NMR}^{*}}>\pi_{1}^{\mathrm{NMV}^{*}}$ if $\Omega_{1}<\Omega<\widehat{\Omega}_{1-1}^{\mathrm{NMM}-\mathrm{NMR}}$, $\pi_{1}^{\mathrm{NMR}^{*}} \geq \pi_{1}^{\mathrm{NMM}^{*}}>\pi_{1}^{\mathrm{NMV}^{*}} \quad$ if $\quad \widehat{\Omega}_{1-1}^{\mathrm{NMM}-\mathrm{NMR}} \leq \Omega$ $<\widehat{\Omega}_{1-1}^{\mathrm{NMM}-\mathrm{NMV}}$, and $\pi_{1}^{\mathrm{NMR}^{*}}>\pi_{1}^{\mathrm{NMV}^{*}} \geq \pi_{1}^{\mathrm{NMM}^{*}}$ if $\widehat{\Omega}_{1-1}^{\mathrm{NMM}-\mathrm{NMV}} \leq \Omega<\Omega_{u}$

(ii) $\pi_{1}^{\mathrm{NRM}^{*}}=\pi_{1}^{\mathrm{NRR}^{*}}>\pi_{1}^{\mathrm{NRV}^{*}}$ regardless of PS and AS

(iii) $\pi_{1}^{\mathrm{NVR}^{*}}=\pi_{1}^{\mathrm{NVM}^{*}}>\pi_{1}^{\mathrm{NVV}^{*}}$ regardless of PS and AS

Here, $\quad \widehat{\Omega}_{1-1}^{\mathrm{NMR}-\mathrm{NMV}}=4-3 \theta^{2} / \theta \widehat{\Omega}_{1-1}^{\mathrm{NMM}-\mathrm{NMV}}=E_{1}$ $+E_{2} \sqrt{6\left(3-\theta^{2}\right)\left(2-\theta^{2}\right)^{3}} / E_{3}$ and $\widehat{\Omega}_{1-1}^{\mathrm{NMM}-\mathrm{NMR}}=K_{1}+K_{2}$ $\sqrt{6\left(6-5 \theta^{2}+\theta^{4}\right)} / K_{3}$. Furthermore, $E_{1}=3072-8576 \theta^{2}+$ $8880 \theta^{4}-3672 \theta^{6}-77 \theta^{8}+558 \theta^{10}-168 \theta^{12}+16 \theta^{14}, \quad E_{2}=4$ $\left(64-148 \theta^{2}+117 \theta^{4}-37 \theta^{6}+4 \theta^{8}\right), \quad E_{3}=4608 \theta-16304 \theta^{3}+$ $24696 \theta^{5}-20679 \theta^{7}+10276 \theta^{9}-3012 \theta^{11}+480 \theta^{13}-32 \theta^{15}$, $K_{1}=10240 \theta-34048 \theta^{3}+45800 \theta^{5}-31756 \theta^{7}+11941 \theta^{9}-$ $2310 \theta^{11}+180 \theta^{13}, K_{2}=\theta\left(1024-2944 \theta^{2}+3204 \theta^{4}-1645 \theta^{6}\right.$ $\left.+397 \theta^{8}-36 \theta^{10}\right)$, and $K_{3}=16384-66560 \theta^{2}+114368 \theta^{4}-$ $106828 \theta^{6}+58138 \theta^{8}-18335 \theta^{10}+3096 \theta^{12}-216 \theta^{14}$.

Under MS game, since $\widehat{\Omega}_{1-2}^{\mathrm{NMR}-\mathrm{NMV}}<\Omega_{1}$ $<\Omega_{u}<\widehat{\Omega}_{1-1}^{\mathrm{NMR}-\mathrm{NMV}}, \pi_{1}^{\mathrm{NMR}^{*}}>\pi_{1}^{\mathrm{NMV}^{*}}$. Moreover, we can then obtain $\widehat{\Omega}_{1-2}^{\mathrm{NMM}-\mathrm{NMV}}<\Omega_{1}<\widehat{\Omega}_{1-1}^{\mathrm{NMM}-\mathrm{NMV}}<\Omega_{u}$ and $\widehat{\Omega}_{1-2}^{\mathrm{NMM}-\mathrm{NMR}}$ $<\Omega_{1}<\Omega_{u}<\widehat{\Omega}_{1-1}^{\mathrm{NMM}-\mathrm{NMR}}$. Therefore, $\pi_{1}^{\mathrm{NMM}^{*}} \geq \pi_{1}^{\mathrm{NMV}^{*}}$ if $\Omega_{1}<\Omega$ $\leq \widehat{\Omega}_{r 1-1}^{\mathrm{NMM}-\mathrm{NMV}}$ and $\pi_{1}^{\mathrm{NMM}^{*}} \geq \pi_{1}^{\mathrm{NMR}^{*}}$ if $\Omega_{1}<\Omega \leq \widehat{\Omega}_{r 1-1}^{\mathrm{NMM}-\mathrm{NMR}}$. Consequently, we have $\pi_{1}^{\mathrm{NMM}^{*}}>\pi_{1}^{\mathrm{NMR}^{*}}>\pi_{1}^{\mathrm{NMV}^{*}}$ if $\Omega_{1}<$ $\Omega<\widehat{\Omega}_{1-1}^{\mathrm{NMM}-\mathrm{NMR}}, \quad \pi_{1}^{\mathrm{NMR}^{*}} \geq \pi_{1}^{\mathrm{NMM}^{*}}>\pi_{1}^{\mathrm{NMV}^{*}} \quad$ if $\widehat{\Omega}_{1-1}^{\mathrm{NMM}-\mathrm{NMR}}$ 
$\leq \Omega<\widehat{\Omega}_{1-1}^{\mathrm{NMM}-\mathrm{NMV}}$ and $\pi_{1}^{\mathrm{NMR}^{*}}>\pi_{1}^{\mathrm{NMV}^{*}} \geq \pi_{1}^{\mathrm{NMM}^{*}}$ if $\widehat{\Omega}_{1-1}^{\mathrm{NMM}-\mathrm{NMV}}$ $\leq \Omega<\Omega_{u}$.

Under RS game, based on the results from Tables 2 and 3, we obtain $\pi_{1}^{\mathrm{NRR}^{*}}=\pi_{1}^{\mathrm{NRM}^{*}}$. Comparing Scenario NRR with Scenario NRV gives us $\widehat{\Omega}_{1-2}^{\mathrm{NRR}-\mathrm{NRV}}<\Omega_{1}<\Omega_{u}<\widehat{\Omega}_{1-1}^{\mathrm{NRR}-\mathrm{NRV}}$, where $\quad \widehat{\Omega}_{1-2}^{\mathrm{NRR}-\mathrm{NRV}}=28 \theta-15 \theta^{3} / 96-119 \theta^{2}+36 \theta^{4} \quad$ and $\widehat{\Omega}_{1-1}^{\mathrm{NRR}-\mathrm{NRV}}=4-3 \theta^{2} / \theta$. Thus, $\pi_{1}^{\mathrm{NRM}^{*}}=\pi_{1}^{\mathrm{NRR}^{*}}>\pi_{1}^{\mathrm{NRV}^{*}}$.

Similarly, we can obtain $\pi_{1}^{\mathrm{NVV}^{*}}<\pi_{1}^{\mathrm{NVR}^{*}}=\pi_{1}^{\mathrm{NVM}^{*}}$ under VN game.

Proof of Proposition 1. The following discussion is also based on the common feasible domain. Similar to the proof of Theorem 1, comparing the equilibrium profits among all game scenarios, we get the following results:

(i) $\pi_{m 1}^{\mathrm{NMM}^{*}}>\pi_{m 1}^{\mathrm{NMR}^{*}}>\pi_{m 1}^{\mathrm{NVM}^{*}}>\pi_{m 1}^{\mathrm{NRR}^{*}} \geq \pi_{m 1}^{\mathrm{NMV}^{*}}>\pi_{m 1}^{\mathrm{NVV}^{*}}>$ $\pi_{m 1}^{\mathrm{NRV}^{*}} \quad$ if $\Omega_{1}<\Omega \leq \widehat{\Omega}_{m 1-1}^{\mathrm{NMV}-\mathrm{NRR}}$, and $\pi_{m 1}^{\mathrm{NMM}^{*}}>$ $\pi_{m 1}^{\mathrm{NMR}^{*}}>\pi_{m 1}^{\mathrm{NMV}^{*}} \geq \pi_{m 1}^{\mathrm{NVM}^{*}}>\pi_{m 1}^{\mathrm{NVV}^{*}}>\pi_{m 1}^{\mathrm{NRR}^{*}}>\pi_{m 1}^{\mathrm{NRV}^{*}}$ if $\widehat{\Omega}_{m 1-1}^{\mathrm{NMV}-\mathrm{NVM}} \leq \Omega<\Omega_{u}$

(ii) $\pi_{r 1}^{\mathrm{NRR}^{*}}>\pi_{r 1}^{\mathrm{NVM}^{*}}>\pi_{r 1}^{\mathrm{NMR}^{*}} \geq \pi_{r 1}^{\mathrm{NMM}^{*}}>\pi_{r 1}^{\mathrm{NRV}^{*}}>\pi_{r 1}^{\mathrm{NVV}^{*}}$ $>\pi_{r 1}^{\mathrm{NMV}^{*}}$ if $\Omega_{1}<\Omega \leq \widehat{\Omega}_{r 1-1}^{\mathrm{NMM}-\mathrm{NMR}}$, and $\pi_{r 1}^{\mathrm{NRR}^{*}}>\pi_{r 1}^{\mathrm{NRV}^{*}}$ $\geq \pi_{r 1}^{\mathrm{NVM}^{*}}>\pi_{r 1}^{\mathrm{NVV}^{*}}>\pi_{r 1}^{\mathrm{NMM}^{*}}>\pi_{r 1}^{\mathrm{NMR}^{*}}>\pi_{r 1}^{\mathrm{NMV}^{*}}$ if $\widehat{\Omega}_{r 1-1}^{\mathrm{NRV}-\mathrm{NVR}}$ $\leq \Omega<\Omega_{u}$

(iii) $\pi_{1}^{\mathrm{NMM}^{*}} \geq \pi_{1}^{\mathrm{NVM}^{*}}>\pi_{1}^{\mathrm{NRR}^{*}}>\pi_{1}^{\mathrm{NVV}^{*}}>\pi_{1}^{\mathrm{NMV}^{*}}$ if $\Omega_{1}<\Omega$ $\leq \widehat{\Omega}_{1-1}^{\mathrm{NVR}-\mathrm{NMM}}, \quad \pi_{1}^{\mathrm{NVM}^{*}}>\pi_{1}^{\mathrm{NVV}^{*}}>\pi_{1}^{\mathrm{NRR}^{*}}>\pi_{1}^{\mathrm{NMM}^{*}}>$ $\pi_{1}^{\mathrm{NMV}^{*}}$ if $\widehat{\Omega}_{1-1}^{\mathrm{NVV}-\mathrm{NRR}}<\Omega \leq \widehat{\Omega}_{1-1}^{\mathrm{NMV}-\mathrm{NMM}}$, and $\pi_{1}^{\mathrm{NVM}^{*}}$ $>\pi_{1}^{\mathrm{NVV}^{*}}>\pi_{1}^{\mathrm{NRR}^{*}}>\pi_{1}^{\mathrm{NMV}^{*}} \geq \pi_{1}^{\mathrm{NMM}^{*}}$ if $\widehat{\Omega}_{1-1}^{\mathrm{NMV}-\mathrm{NMM}}$ $\leq \Omega<\Omega_{u}$

Here, $\pi_{m 1}^{\mathrm{NVM}^{*}}=\pi_{m 1}^{\mathrm{NVR}^{*}}, \quad \pi_{m 1}^{\mathrm{NRR}^{*}}=\pi_{m 1}^{\mathrm{NRM}^{*}}, \quad \pi_{r 1}^{\mathrm{NRR}^{*}}=\pi_{r 1}^{\mathrm{NRM}^{*}}$, $\pi_{r 1}^{\mathrm{NVM}^{*}}=\pi_{r 1}^{\mathrm{NVR}^{*}}, \quad \pi_{1}^{\mathrm{NVM}^{*}}=\pi_{1}^{\mathrm{NVR}^{*}}, \quad \pi_{1}^{\mathrm{NRR}^{*}}=\pi_{1}^{\mathrm{NMR}^{*}}=\pi_{1}^{\mathrm{NRM}^{*}}$, $\pi_{1}^{\mathrm{NMV}^{*}}=\pi_{1}^{\mathrm{NRV}^{*}}, \widehat{\Omega}_{m 1-1}^{\mathrm{NMV}-\mathrm{NRR}}=480 \theta-872 \theta^{3}+531 \theta^{5}-108 \theta^{7}$ $+3 \sqrt{2} \theta\left(32-66 \theta^{2}+43 \theta^{4}-9 \theta^{6}\right) / 1152-2784 \theta^{2}+2545 \theta^{4}-$

$1044 \theta^{6}+162 \theta^{8}, \widehat{\Omega}_{r 1-1}^{\mathrm{NMM}-\mathrm{NMR}}=\theta\left(16-19 \theta^{2}+5 \theta^{4}\right) / 32-58 \theta^{2}$ $+34 \theta^{4}-6 \theta^{6}, \widehat{\Omega}_{m 1-1}^{\mathrm{NMV}-\mathrm{NVM}}=\widehat{\Omega}_{r 1-1}^{\mathrm{NRV}-\mathrm{NVR}}=2 \theta-\theta^{3}+\sqrt{2} \theta(1-$ $\left.\theta^{2}\right) \quad / 2-\theta^{4}, \quad \widehat{\Omega}_{1-1}^{\mathrm{NVR}-\mathrm{NMM}}=L_{1}+L_{2} \sqrt{\left(3-\theta^{2}\right)\left(2-\theta^{2}\right)^{3}} / L_{3}$, $\widehat{\Omega}_{1-1}^{\mathrm{NVV}-\mathrm{NMM}}=M_{1}+M_{2} \sqrt{6-5 \theta^{2}+\theta^{4}} / M_{3}$, and $\widehat{\Omega}_{1-1}^{\mathrm{NVV}-\mathrm{NRR}}=$ $564 \theta-1159 \theta^{3}+798 \theta^{5}-180 \theta^{7}+\sqrt{6} \theta\left(144-289 \theta^{2}+181 \theta^{4}-\right.$ $\left.36 \theta^{6}\right) / 720-2040 \theta^{2}+2279 \theta^{4}-1152 \theta^{6}+216 \theta^{8}$. Furthermore, $\quad L_{1}=2560 \theta-7488 \theta^{3}+7680 \theta^{5}-2480 \theta^{7}-996 \theta^{9}+$ $969 \theta^{11}-260 \theta^{13}+24 \theta^{15}, \quad L_{2}=6 \theta^{3}\left(64-148 \theta^{2}+117 \theta^{4}-37\right.$ $\left.\theta^{6}+4 \theta^{8}\right), \quad L_{3}=10240-44544 \theta^{2}+80544 \theta^{4}-78032 \theta^{6}+$ $43560 \theta^{8}-13980 \theta^{10}+2389 \theta^{12}-168 \theta^{14}, \quad M_{1}=4512 \theta-$ $14420 \theta^{3}+18672 \theta^{5}-12481 \theta^{7}+4532 \theta^{9}-848 \theta^{11}+64 \theta^{13}$, $M_{2}=2 \theta\left(576-1588 \theta^{2}+1645 \theta^{4}-801 \theta^{6}+184 \theta^{8}-16 \theta^{10}\right)$, and $M_{3}=5760-22368 \theta^{2}+37114 \theta^{4}-33739 \theta^{6}+17972 \theta^{8}-$ $5572 \theta^{10}+928 \theta^{12}-64 \theta^{14}$.

For the manufacturer 1, based on the proofs of Lemma 2, we find the following results:

$$
\Delta \pi_{m 1}^{\mathrm{NMV}^{*}-\mathrm{NRR}^{*}}(\Omega, \theta)<0 \quad \text { if } \quad \Omega_{\mathrm{l}}<\Omega<\widehat{\Omega}_{m 1-1}^{\mathrm{NMV}-\mathrm{NRR}},
$$
$\Delta \pi_{m 1}^{\mathrm{NMV}^{*}-\mathrm{NVM}^{*}}(\Omega, \theta)<0 \quad$ if $\quad \Omega_{1}<\Omega<\widehat{\Omega}_{\mathrm{m} 1-1}^{\mathrm{NMV}-\mathrm{NVM}}$ and
$\Delta \pi_{m 1}^{\mathrm{NVV}^{*}-\mathrm{NRR}^{*}}(\Omega, \theta)<0$ if $\Omega_{1}<\Omega<\widehat{\Omega}_{m 1-1}^{\mathrm{NVV}-\mathrm{NRR}}$. Meantime, we can show that $\Delta \pi_{m 1}^{\mathrm{NMR}^{*}-\mathrm{NVM}^{*}}(\Omega, \theta)>0$, $\Delta \pi_{m 1}^{\mathrm{NVM}^{*}-\mathrm{NRR}^{*}}(\Omega, \theta)>0, \quad \Delta \pi_{m 1}^{\mathrm{NMV}^{*}-\mathrm{NVV}^{*}}(\Omega, \theta)>0, \quad$ and $\Delta \pi_{m 1}^{\mathrm{NVV}^{*}-\mathrm{NRV}^{*}}(\Omega, \theta)>0 . \quad$ Furthermore, $\quad \widehat{\Omega}_{m 1-1}^{\mathrm{NMV}-\mathrm{NVM}}$ $>\widehat{\Omega}_{m 1-1}^{\mathrm{NMV}-\mathrm{NRR}}>\widehat{\Omega}_{m 1-1}^{\mathrm{NMV}-\mathrm{NRR}}$. Consequently, we can infer the result of Part (i).

Similar to the above proofs, we provide results for the retailer 1 in the following.

Comparing the retailer 1's profits under different price leadership scenarios, we obtain $\Delta \pi_{r l}^{\mathrm{NRV}^{*}-\mathrm{NVR}^{*}}(\Omega, \theta)<0$ if $\Omega_{1}<\Omega<\widehat{\Omega}_{r 1-1}^{\mathrm{NRV}-\mathrm{NVR}}, \quad \Delta \pi_{r 1}^{\mathrm{NRV}^{*}-\mathrm{NMM}^{*}}(\Omega, \theta)<0$ if $\Omega_{1}<\Omega<$ $\widehat{\Omega}_{r 1-1}^{\mathrm{NRV}-\mathrm{NMM}^{2}}, \Delta \pi_{r 1}^{\mathrm{NRV}^{*}-\mathrm{NMR}^{*}}(\Omega, \theta)<0$ if $\Omega_{\mathrm{l}}<\Omega<\widehat{\Omega}_{r 1-1}^{\mathrm{NRV}-\mathrm{NMR}}$, $\Delta \pi_{r 1}^{\mathrm{NVV}^{*}-\mathrm{NMM}^{*}}(\Omega, \theta)<0 \quad$ if $\quad \Omega_{1}<\Omega<\widehat{\Omega}_{r 1-1}^{\mathrm{NRV}-\mathrm{NMR}}$, and $\Delta \pi_{r 1}^{\mathrm{NVV}^{*}-\mathrm{NMR}^{*}}(\Omega, \theta)<0$ if $\Omega_{1}<\Omega<\widehat{\Omega}_{r 1-1}^{\mathrm{NVV}-\mathrm{NMR}}$. Meantime, $\Delta \pi_{r 1}^{\mathrm{NRV}^{*}-\mathrm{NVV}^{*}}(\Omega, \theta)>0, \quad \Delta \pi_{r 1}^{\mathrm{NVV}^{*}-\mathrm{NMV}^{*}}(\Omega, \theta)>0$, $\Delta \pi_{r 1}^{\mathrm{NVR}^{*}-\mathrm{NMR}^{*}}(\Omega, \theta)>0, \Delta \pi_{r 1}^{\mathrm{NVR}^{*}-\mathrm{NMM}^{*}}(\Omega, \theta)>0$. Moreover, $\widehat{\Omega}_{r 1-1}^{\mathrm{NRV}-\mathrm{NVR}}>\widehat{\Omega}_{r 1-1}^{\mathrm{NVV}-\mathrm{NMR}}>\widehat{\Omega}_{r 1-1}^{\mathrm{NVV}-\mathrm{NMM}}>\widehat{\Omega}_{r 1-1}^{\mathrm{NRV}-\mathrm{NMM}}>\widehat{\Omega}_{r 1-1}^{\mathrm{NRV}-\mathrm{NMR}}$ $>\widehat{\Omega}_{r 1-1}^{\text {NMM-NMR }}$. From the above relationships, we have the result of Part (ii).

Similar analysis applies to the channel 1, using the contour plot, we can graphically show that (as shown in Figure 11) $\widehat{\Omega}_{1-1}^{\mathrm{NRV}-\mathrm{NVR}}>\widehat{\Omega}_{1-1}^{\mathrm{NVV}-\mathrm{NMR}}>\widehat{\Omega}_{1-1}^{\mathrm{NVV}-\mathrm{NMM}}>$ $\widehat{\Omega}_{1-1}^{\mathrm{NRV}-\mathrm{NMM}}>\widehat{\Omega}_{1-1}^{\mathrm{NRV}-\mathrm{NMR}}>\widehat{\Omega}_{1-1}^{\mathrm{NMM}-\mathrm{NMR}}$. Methods similar to those described earlier, we obtain the result of Part (iii).

Proof of Theorem 2. Theorem 2 is a direct result from Proposition 1.

Proof of Lemma 3. This Lemma's proof is similar to that of Lemma 2. More specifically, we first provide the comparison for the manufacturer 1 and the retailer 1 and then extend our proof to the channel 1 . Comparing the manufacturer 1 ' profits, we obtain the following:

(i) $\pi_{m 1}^{\mathrm{MM}^{*}}=\pi_{m 1}^{\mathrm{MR}^{*}} \geq \pi_{m 1}^{\mathrm{MV}^{*}}$ if $1<\Omega \leq \widehat{\Omega}_{m 1-1}^{\mathrm{MM}-\mathrm{MV}}$

(ii) $\pi_{m 1}^{\mathrm{RM}^{*}}=\pi_{m 1}^{\mathrm{RR}} \geq \pi_{m 1}^{\mathrm{RV}^{*}}$ if $1<\Omega \leq \widehat{\Omega}_{m 1-1}^{\mathrm{MM}-\mathrm{MV}}$

(iii) $\pi_{m 1}^{\mathrm{VM}^{*}}=\pi_{m 1}^{\mathrm{VR}^{*}} \geq \pi_{m 1}^{\mathrm{VV}^{*}}$ if $1<\Omega \leq \widehat{\Omega}_{m 1-1}^{\mathrm{MM}-\mathrm{MV}}$

Here, $\widehat{\Omega}_{m 1-1}^{\mathrm{MM}-\mathrm{MV}}=\widehat{\Omega}_{m 1-1}^{\mathrm{RR}-\mathrm{RV}}=\widehat{\Omega}_{m 1-1}^{\mathrm{VM}-\mathrm{VR}}=48-20 \theta^{2}-$ $48 \theta^{4}+27 \theta^{6}+12\left(2-3 \theta^{2}+\theta^{4}\right) \quad \sqrt{\left(4-3 \theta^{2}\right)} / 240 \theta-500 \theta^{3}+$ $348 \theta^{5}-81 \theta^{7}$.

Under MS game, since $\widehat{\Omega}_{1-2}^{\mathrm{MM}-\mathrm{MV}}<1<\widehat{\Omega}_{1-\mathrm{I}_{\mathrm{MM}} \mathrm{MV}}^{\mathrm{MM}-\mathrm{MV}} \Omega_{u}$. Thus, we have $\pi_{m 1}^{\mathrm{MM}^{*}}=\pi_{m 1}^{\mathrm{MR}^{*}} \geq \pi_{m 1}^{\mathrm{MV}}$ if $1<\Omega<\Omega_{m 1-1}$. A similar situation arises with regards to RS game, because $\widehat{\Omega}_{m 1-1}^{\mathrm{RR}-\mathrm{RV}}=\widehat{\Omega}_{m 1-1}^{\mathrm{MM}-\mathrm{MV}}$ and $\widehat{\Omega}_{m 1-2}^{\mathrm{RR}-\mathrm{RV}}=\widehat{\Omega}_{m 1-2}^{\mathrm{MM}-\mathrm{MV}}$, we obtain $\pi_{m 1}^{\mathrm{RM}^{*}}=\pi_{m 1}^{\mathrm{RR}} \geq \pi_{m 1}^{\mathrm{RV}}$ if $1<\Omega \leq \widehat{\Omega}_{m 1-1}^{\mathrm{MM}-\mathrm{MV}}$. Similarly, considering VN game, we have $\pi_{m 1}^{\mathrm{VM}^{*}}=\pi_{m 1}^{\mathrm{VR}^{*}} \geq \pi_{m 1}^{\mathrm{VV}^{*}}$ if $1<\Omega \leq \widehat{\Omega}_{m 1-1}^{\mathrm{MM}-\mathrm{MV}}$.

Similar to the proof in Lemma 2, we obtain the following results for retailer 1 :

(i) $\pi_{r 1}^{\mathrm{MM}^{*}}=\pi_{r 1}^{\mathrm{MR}^{*}} \geq \pi_{r 1}^{\mathrm{MV}^{*}}$ if $1<\Omega \leq \widehat{\Omega}_{m 1-1}^{\mathrm{MM}-\mathrm{MV}}$

(ii) $\pi_{r 1}^{\mathrm{RM}^{*}}=\pi_{r 1}^{\mathrm{RR}^{*}} \geq \pi_{r 1}^{\mathrm{RV}^{*}}$ if $1<\Omega \leq \widehat{\Omega}_{m 1-1}^{\mathrm{MM}-\mathrm{MV}}$ 
(iii) $\pi_{r 1}^{\mathrm{VM}^{*}}=\pi_{r 1}^{\mathrm{VR}^{*}} \geq \pi_{r 1}^{\mathrm{VV}^{*}}$ if $1<\Omega \leq \widehat{\Omega}_{m 1-1}^{\mathrm{MM}-\mathrm{MV}}$ Here, $\widehat{\Omega}_{r 1-1}^{\mathrm{MM}-\mathrm{MV}}=\widehat{\Omega}_{r 1-1}^{\mathrm{RR}-\mathrm{RV}}=\widehat{\Omega}_{r 1-1}^{\mathrm{VM}-\mathrm{VR}}=\widehat{\Omega}_{m 1-1}^{\mathrm{MM}-\mathrm{MV}}$.

Under MS game, since $\widehat{\Omega}_{r 1-1}^{\mathrm{MM}-\mathrm{MV}}=\widehat{\Omega}_{m 1-1}^{\mathrm{MM}-\mathrm{MV}}$ and $\widehat{\Omega}_{r 1-2}^{\mathrm{MM}-\mathrm{MV}}=\widehat{\Omega}_{m 1-2}^{\mathrm{MM}-\mathrm{MV}}$, we get the similar results, that is, $\pi_{r 1}^{\mathrm{VM}^{*}}=\pi_{r 1}^{\mathrm{VR}^{*}} \geq \pi_{r 1}^{\mathrm{VV}^{*}}$ if $1<\Omega \leq \widehat{\Omega}_{m 1-1}^{\mathrm{MM}-\mathrm{MV}}$. Similar results arise in $\mathrm{RS}$ and $\mathrm{VN}$ games, that is, $\pi_{r 1}^{\mathrm{RV}^{*}} \leq \pi_{r 1}^{\mathrm{RM}^{*}}=\pi_{r 1}^{\mathrm{RR}^{*}}$ if $1<\Omega \leq \widehat{\Omega}_{m 1-1}^{\mathrm{MM}-\mathrm{MV}}$ and $\quad \pi_{r 1}^{\mathrm{VM}^{*}}=\pi_{r 1}^{\mathrm{VR}^{*}} \geq \pi_{r 1}^{\mathrm{VV}^{*}} \quad$ if $\quad 1<\Omega$ $\leq \widehat{\Omega}_{m 1-1}^{\mathrm{MM}-\mathrm{MV}}$. This is because $\widehat{\Omega}_{r 1-1}^{\mathrm{RR}-\mathrm{RV}}=\widehat{\Omega}_{r 1-1}^{\mathrm{VM}-\mathrm{VR}}=\widehat{\Omega}_{m 1-1}^{\mathrm{MM}-\mathrm{MV}}$.

Lastly, for the channel $1, \pi_{r 1}^{\mathrm{MM}^{*}}=\pi_{r 1}^{\mathrm{MR}^{*}}, \pi_{m 1}^{\mathrm{MM}^{*}}=\pi_{m 1}^{\mathrm{MR}^{*}}$, $\pi_{r 1}^{\mathrm{RR}^{*}}=\pi_{r 1}^{\mathrm{RM}^{*}}, \pi_{m 1}^{\mathrm{RR}^{*}}=\pi_{m 1}^{\mathrm{RM}^{*}}, \pi_{r 1}^{\mathrm{VM}^{*}}=\pi_{r 1}^{\mathrm{VR}^{*}}$, and $\pi_{m 1}^{\mathrm{VM}^{*}}=\pi_{m 1}^{\mathrm{VR}^{*}}$, so the channel has the properties with channel members. Here, we omit it for brevity.

Proof of Theorem 3. Similar to the proof of Theorem 2, we have the following results:

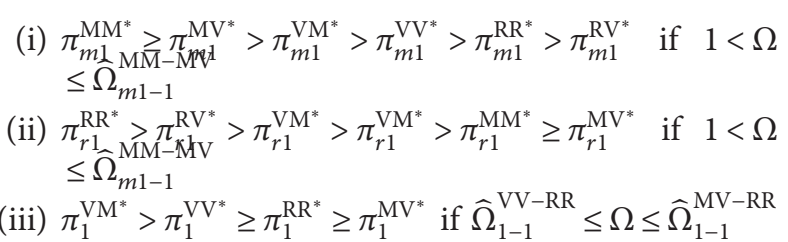

Here, $\quad \pi_{m 1}^{\mathrm{MM}^{*}}=\pi_{m 1}^{\mathrm{MR}^{*}}, \quad \pi_{m 1}^{\mathrm{VM}^{*}}=\pi_{m 1}^{\mathrm{VR}^{*}}, \quad \pi_{m 1}^{\mathrm{RR}^{*}}=\pi_{m 1}^{\mathrm{RM}^{*}}$, $\pi_{r 1}^{\mathrm{RR}^{*}}=\pi_{r 1}^{\mathrm{RM}^{*}}, \quad \pi_{r 1}^{\mathrm{VM}^{*}}=\pi_{r 1}^{\mathrm{VR}^{*}}, \quad \pi_{r 1}^{\mathrm{MM}^{*}}=\pi_{r 1}^{\mathrm{MR}^{*}}, \quad \pi_{1}^{\mathrm{VM}^{*}}=\pi_{1}^{\mathrm{VR}^{*}}$, $\pi_{1}^{\mathrm{RR}^{*}}=\pi_{1}^{\mathrm{MM}^{*}}=\pi_{1}^{\mathrm{MR}^{*}}=\pi_{1}^{\mathrm{RM}^{*}}$, and $\pi_{1}^{\mathrm{MV}^{*}}=\pi_{1}^{\mathrm{RV}^{*}}$. Moreover, $\widehat{\Omega}_{m 1-1}^{\mathrm{MM}-\mathrm{MV}}=\widehat{\Omega}_{r 1-1}^{\mathrm{MM}-\mathrm{MV}}=\widehat{\Omega}_{1-1}^{\mathrm{MM}-\mathrm{MV}}=48-20 \theta^{2}-48 \theta^{4}+27 \theta^{6}$ $+12\left(2-3 \theta^{2}+\theta^{4}\right) \sqrt{\left(4-3 \theta^{2}\right)} / 240 \theta-500 \theta^{3}+348 \theta^{5}-81 \theta^{7}$.

For the manufacturer 1 , we have $\Delta \pi_{m 1}^{\mathrm{MM}^{*}-\mathrm{RR}^{*}}(\Omega, \theta)=\left(\theta-4 \Omega+3 \quad \theta^{2} \Omega\right)^{2} / 64\left(4-7 \theta^{2}+3 \theta^{4}\right)$ $>0, \Delta \pi_{m 1}^{\mathrm{MM}^{*}-\mathrm{VM}^{*}}(\Omega, \theta)=\left(\theta-4 \Omega+3 \quad \theta^{2} \Omega\right)^{2} / 288\left(4-7 \theta^{2}\right.$ $\left.+3 \theta^{4}\right)>0, \Delta \pi_{m 1}^{\mathrm{RR}}-\mathrm{VM}^{*}(\Omega, \theta)=-7\left(\theta-4 \Omega+3 \theta^{2} \Omega\right)^{2} / 576(4$ $\left.-7 \theta^{2}+3 \theta^{4}\right)<0$, and $\Delta \pi_{m 1}^{\mathrm{RV}^{*}-\mathrm{VV}^{*}}(\Omega, \theta)=-7\left(\theta-3 \Omega+2 \theta^{2}\right.$ $\Omega)^{2} / 432\left(3-5 \theta^{2}+2 \theta^{4}\right)<0 . \quad$ Meantime, $\quad \widehat{\Omega}_{m 1-2}^{\mathrm{MV}-\mathrm{VM}}<\Omega_{1}$ $<\widehat{\Omega}_{m 1-1}^{\mathrm{MV}-\mathrm{VM}}<1<\Omega_{u}$ and $\partial^{2} \Delta \pi_{m 1}^{\mathrm{MV}^{*}-\mathrm{VM}^{*}} / \partial \Omega^{2}>0$; hence, $\pi_{m 1}^{\mathrm{MV}^{*}}>\pi_{m 1}^{\mathrm{VM}^{*}}$. Similarly, it is easy to see that $\pi_{m 1}^{\mathrm{VV}^{*}}>\pi_{m 1}^{\mathrm{RR}}$ and $\pi_{m 1}^{\mathrm{VM}^{*}}>\pi_{m 1}^{\mathrm{RV}}$. Therefore, we infer that $\pi_{m 1}^{\mathrm{MM}^{*}} \geq \pi_{m 1}^{\mathrm{MV}}>\pi_{m 1}^{\mathrm{VM}^{*}}>\pi_{m 1}^{\mathrm{VV}^{*}}>\pi_{m 1}^{\mathrm{RR}^{*}}>\pi_{m 1}^{\mathrm{RV}^{*}} \quad$ if $\quad 1<\Omega$ $\leq \widehat{\Omega}_{m 1-1}^{\mathrm{MM}-\mathrm{MV}}$ from the above analysis.

Similar results arise for the retailer 1 , which are omitted here for brevity. For the channel 1, we can easily get the results, $\quad \pi_{1}^{\mathrm{VM}^{*}}=\pi_{1}^{\mathrm{VR}^{*}}, \quad \pi_{1}^{\mathrm{RR}^{*}}=\pi_{1}^{\mathrm{MM}^{*}}=\pi_{1}^{\mathrm{MR}^{*}}=\pi_{1}^{\mathrm{RM}^{*}}, \quad$ and $\pi_{1}^{\mathrm{MV}^{*}}=\pi_{1}^{\mathrm{RV}}$, from Tables 6 and 7. A similar process obtains $\pi_{1}^{\mathrm{VM}^{*}}>\pi_{1}^{\mathrm{VV}^{*}} \geq \pi_{1}^{\mathrm{RR}^{*}} \geq \pi_{1}^{\mathrm{MV}{ }^{*}}$ if $\widehat{\Omega}_{1-1}^{\mathrm{VV}-\mathrm{RR}} \leq \Omega \leq \widehat{\Omega}_{1-1}^{\mathrm{MV}-\mathrm{RR}}$.

Proof of Corollaries 1-2. The Proof is straightforward; here, we omit it for brevity.

Proof of Theorems 4-5. Given the symmetric setting, the problem is much easier to solve and is hence omitted.

Proof of Lemma 4. We first compare the equilibrium wholesale prices under MS game, RS game, and VN game, respectively. Then, we explore the similar problems for the retail prices. Given a price leadership situation, we have the following results:

(i) $w_{1}^{\mathrm{NMM}^{*}}>w_{1}^{\mathrm{NMR}^{*}}>w_{1}^{\mathrm{NMV}^{*}}$ for any $\theta \in(0,1)$

(ii) $w_{1}^{\mathrm{NRR}^{*}}=w_{1}^{\mathrm{NRM}^{*}}>w_{1}^{\mathrm{NRV}^{*}}$ for any $\theta \in(0,1)$

(iii) $w_{1}^{\mathrm{NVR}^{*}}=w_{1}^{\mathrm{NVM}^{*}}>w_{1}^{\mathrm{NVV}^{*}}$ for any $\theta \in(0,1)$

We first prove the wholesale price Under MS game. Since $w_{1}^{\mathrm{NMM}^{*}}-w_{1}^{\mathrm{NMR}^{*}}=(1-\theta) \theta^{2} A_{2} /(4-3 \theta)\left(4-\theta-2 \theta^{2}\right)>0$ and $w_{1}^{\mathrm{NMR}^{*}}-w_{1}^{\mathrm{NMV}^{*}}=(1-\theta) \theta / 3(4-3 \theta)\left(2-\theta^{2}\right)>0$, we can thus have $w_{1}^{\mathrm{NMM}^{*}}>w_{1}^{\mathrm{NMR}^{*}}>w_{1}^{\mathrm{NMV}^{*}}$. Comparing the wholesale price Under RS game, we have $w_{1}^{\mathrm{NRR}^{*}}=w_{1}^{\mathrm{NRM}^{*}}$ and $\quad w_{1}^{\mathrm{NRR}^{*}}-w_{1}^{\mathrm{NRV}^{*}}=\theta(1-\theta) / 6(4-3 \theta)\left(2-\theta^{2}\right)>0$. Therefore, we prove that $w_{1}^{\mathrm{NRR}^{*}}=w_{1}^{\mathrm{NRM}^{*}}>w_{1}^{\mathrm{NRV}^{*}}$. Similarly, under VN game, we have $w_{1}^{\mathrm{NVR}^{*}}=w_{1}^{\mathrm{NVM}^{*}}>w_{1}^{\mathrm{NVV}^{*}}$ due to $w_{1}^{\mathrm{NVR}^{*}}=w_{1}^{\mathrm{NVM}^{*}}$ and $w_{1}^{\mathrm{NVV}^{*}}-w_{1}^{\mathrm{NVR}^{*}}=-(1-\theta) \theta / 6$ $(3-2 \theta)\left(2-\theta^{2}\right)<0$.

A similar process obtains the results for the retail price under MS game, RS game, and VN game.

(i) $p_{1}^{\mathrm{NMM}^{*}}>p_{1}^{\mathrm{NMR}^{*}}>p_{1}^{\mathrm{NMV}^{*}}$ for any $\theta \in(0,1)$

(ii) $p_{1}^{\mathrm{NRR}^{*}}=p_{1}^{\mathrm{NRM}^{*}}>p_{1}^{\mathrm{NRV}^{*}}$ for any $\theta \in(0,1)$

(iii) $p_{1}^{\mathrm{NVR}^{*}}=p_{1}^{\mathrm{NVM}^{*}}>p_{1}^{\mathrm{NVV}^{*}}$ for any $\theta \in(0,1)$

Similar to the proofs for the wholesale prices, under MS game, it is easy to see that $p_{1}^{\mathrm{NMM}^{*}}-p_{1}^{\mathrm{NMR}^{*}}=(1-\theta) \theta^{2} /(2-$ $\theta)(4-3 \theta)\left(4-\theta-2 \theta^{2}\right)>0$ and $p_{1}^{\mathrm{NMR}^{*}}-p_{1}^{\mathrm{NMV}^{*}}=$ $(1-\theta) \theta / 2(4-3 \theta)\left(2-\theta^{2}\right)>0$. Hence, we have $p_{1}^{\mathrm{NMM}^{*}}>p_{1}^{\mathrm{NMR}^{*}}>p_{1}^{\mathrm{NMV}^{*}}$. Furthermore, since $p_{1}^{\mathrm{NRR}^{*}}$ $=p_{1}^{\mathrm{NRM}^{*}}, \quad p_{1}^{\mathrm{NVR}^{*}}=p_{1}^{\mathrm{NVM}^{*}}, \quad p_{1}^{\mathrm{NRR}^{*}}-p_{1}^{\mathrm{NRV}^{*}}=(1-\theta) \theta / 2$ $(4-3 \theta)\left(2-\theta^{2}\right)>0, \quad$ and $p_{1}^{\mathrm{NVV}^{*}}-p_{1}^{\mathrm{NVR}^{*}}=-(1-\theta) \theta / 3$ $(3-2 \theta)\left(2-\theta^{2}\right)<0$, we have $p_{1}^{\mathrm{NRR}^{*}}=p_{1}^{\mathrm{NRM}^{*}}>p_{1}^{\mathrm{NRV}^{*}}$ and $p_{1}^{\mathrm{NVR}^{*}}=p_{1}^{\mathrm{NVM}^{*}}>p_{1}^{\mathrm{NVV}^{*}}$.

Note that the values for Manufacturer 2 can be obtained by replacing every 1 with 2 and vice versa. Other results are omitted for brevity.

Proof of Theorem 6. Based on the proofs of Lemma 4, it is easy to find that, given a price leadership situation, the wholesale price 1 is higher in Scenario NMM, Scenario NRR (NRM), or Scenario NVM (NVR), while lower in Scenario NMV, Scenario NRV, or NVV. Therefore, one can easily find the wholesale price 1 is highest in Scenario NMM and lowest in Scenario NRV. Similarly, we can obtain the results for retail price under all price leadership scenarios.

Proof of Lemma 5. Comparing the equilibrium demands across MS game, RS game, and VN game, we find the following results:

(i) $D_{1}^{\mathrm{NMR}^{*}}>D_{1}^{\mathrm{NMM}^{*}}>D_{1}^{\mathrm{NMV}^{*}}$ for any $\theta \in(0,1)$

(ii) $D_{1}^{\mathrm{NRR}^{*}}=D_{1}^{\mathrm{NRM}^{*}}>D_{1}^{\mathrm{NRV}^{*}}$ for any $\theta \in(0,1)$

(iii) $D_{1}^{\mathrm{NVR}^{*}}=D_{1}^{\mathrm{NVM}^{*}}>D_{1}^{\mathrm{NVV}^{*}}$ for any $\theta \in(0,1)$

Demand is assumed to be decreasing in its price, and increasing in the competing channel's price, and combined with the equilibrium retail prices discussed in the previous subsection. It is easy to prove Lemma 2. For instance, the price gap between two channels in Scenario NRM is larger than that in Scenario NRV (NVV) (e.g., 
$\left.p_{2}^{\mathrm{NRM}}-P_{1}^{\mathrm{NRM}}>p_{2}^{\mathrm{NRV}}-P_{1}^{\mathrm{NRV}}\right)$; thus, Scenario NRM always results in larger demand than that in Scenario NRV. Moreover, the demand levels are equal under Scenario NRR/ NRM (e.g., $\quad D_{1}^{\mathrm{NRR}}=D_{1}^{\mathrm{NRM}}$ ). Here, the details are omitted.

Proof of Theorem 7. Similar to the proof in Theorem 6, it is easy to see that the demand 1 is smallest in Scenario NMV/ NRV and largest in Scenario NVM/NVR; thus, the details are skipped.

\section{Data Availability}

The data used to support the findings of this study are available from the corresponding author upon request.

\section{Conflicts of Interest}

The authors declare that they have no conflicts of interest.

\section{References}

[1] S. C. Choi, "Price competition in a channel structure with a common retailer," Marketing Science, vol. 10, no. 1, pp. 271-296, 1991.

[2] G. Cai, Z. G. Zhang, and M. Zhang, "Game theoretical perspectives on dual-channel supply chain competition with price discounts and pricing schemes," International Journal of Production Economics, vol. 117, no. 1, pp. 80-96, 2009.

[3] H. Chung and E. Lee, "Asymmetric relationships with symmetric suppliers: strategic choice of supply chain price leadership in a competitive market," European Journal of Operational Research, vol. 259, no. 2, pp. 564-575, 2017.

[4] E. Lee and R. Staelin, "Vertical strategic interaction: implications for channel pricing strategy," Marketing Science, vol. 16, no. 3, pp. 185-207, 1997.

[5] J. Wei, J. Zhao, and Y. Li, "Pricing decisions for complementary products with firms' different market powers," European Journal of Operational Research, vol. 224, no. 3, pp. 507-519, 2013.

[6] T. W. McGuire and R. Staelin, "An industry equilibrium analysis of downstream vertical integration," Marketing Science, vol. 2, no. 2, pp. 161-191, 1983.

[7] S.-L. Yang and Y.-W. Zhou, "Two-echelon supply chain models: considering duopolistic retailers' different competitive behaviors," International Journal of Production Economics, vol. 103, no. 1, pp. 104-116, 2006.

[8] R. Zhang, B. Liu, and W. Wang, "Pricing decisions in a dual channels system with different power structures," Economic Modelling, vol. 29, no. 2, pp. 523-533, 2012.

[9] N. C. P. Edirisinghe, B. Bichescu, and X. Shi, "Equilibrium analysis of supply chain structures under power imbalance," European Journal of Operational Research, vol. 214, no. 3, pp. 568-578, 2011.

[10] M. M. Seyedesfahani, M. Biazaran, and M. Gharakhani, “A game theoretic approach to coordinate pricing and vertical co-op advertising in manufacturer-retailer supply chains," European Journal of Operational Research, vol. 211, no. 2, pp. 263-273, 2011.

[11] J. Chaeb and M. Rasti Barzoki, "Coordination via cooperative advertising and pricing in a manufacturer-retailer supply chain," Computers \& Industrial Engineering, vol. 99, pp. 112-123, 2016.
[12] R. Shi, J. Zhang, and J. Ru, "Impacts of power structure on supply chains with uncertain demand," Production \& Operations Management, vol. 22, no. 5, pp. 1232-1249, 2013.

[13] X. Chen and X. Wang, "Free or bundled: channel selection decisions under different power structures," Omega, vol. 53, pp. 11-20, 2015.

[14] X. Chen, X. Wang, and H. K. Chan, "Manufacturer and retailer coordination for environmental and economic competitiveness: a power perspective," Transportation Research Part E: Logistics and Transportation Review, vol. 97, pp. 268-281, 2017.

[15] J. Zhao, J. Wei, and Y. Li, "Pricing decisions for substitutable products in a two-echelon supply chain with firms' different channel powers," International Journal of Production Economics, vol. 153, pp. 243-252, 2014.

[16] J. Zhao, X. Hou, Y. Guo, and J. Wei, "Pricing policies for complementary products in a dual-channel supply chain," Applied Mathematical Modelling, vol. 49, pp. 437-451, 2017.

[17] S. A. Raza and S. M. Govindaluri, "Pricing strategies in a dualchannel green supply chain with cannibalization and risk aversion," Operations Research Perspectives, vol. 6, pp. 100$118,2019$.

[18] A. P. Jeuland and S. Steven, "Managing channel profits," Marketing Science, vol. 2, pp. 239-272, 1983.

[19] C.-H. Wu, C.-W. Chen, and C.-C. Hsieh, "Competitive pricing decisions in a two-echelon supply chain with horizontal and vertical competition," International Journal of Production Economics, vol. 135, no. 1, pp. 265-274, 2012.

[20] J. Ma and Q. Li, "The complex dynamics of BertrandStackelberg pricing models in a risk-averse supply chain," Discrete Dynamics in Nature and Society, vol. 2014, pp. 1-14, 2014.

[21] H. Huang, H. Ke, and L. Wang, "Equilibrium analysis of pricing competition and cooperation in supply chain with one common manufacturer and duopoly retailers," International Journal of Production Economics, vol. 178, pp. 12-21, 2016.

[22] Z. Luo, X. Chen, J. Chen, and X. Wang, "Optimal pricing policies for differentiated brands under different supply chain power structures," European Journal of Operational Research, vol. 259, no. 2, pp. 437-451, 2017.

[23] L. Wang, H. Song, and Y. Wang, "Pricing and service decisions of complementary products in a dual-channel supply chain," Computers \& Industrial Engineering, vol. 105, pp. 223-233, 2017.

[24] T. Li, R. Zhang, and B. Liu, "Pricing decisions of competing supply chains under power imbalance structures," Computers \& Industrial Engineering, vol. 125, pp. 695-707, 2018.

[25] B. Liu, T. Li, and S. B. Tsai, "Low carbon strategy analysis of competing supply chains with different power structures," Sustainability, vol. 9, no. 5, p. 835, 2017.

[26] V. Kadiyali, P. Chintagunta, and N. Vilcassim, "Manufacturer-retailer channel interactions and implications for channel power: an empirical investigation of pricing in a local market," Marketing Science, vol. 19, no. 2, pp. 127-148, 2000.

[27] W. E. Kuiper and M. T. G. Meulenberg, "Price leadership within a marketing channel: a cointegration study," International Journal of Research in Marketing, vol. 21, no. 2, pp. 137-158, 2004.

[28] R. M. S. Costa and P. Pavone, "Diachronic biodiversity analysis of a metropolitan area in the Mediterranean region," Acta Horticulturae, vol. 1215, no. 1215, pp. 49-52, 2018.

[29] T. Boyaci and G. Gallego, "Supply chain coordination in a market with customer service competition," Production and Operations Management, vol. 13, no. 1, pp. 3-22, 2004. 
[30] A. Y. Ha and S. Tong, "Contracting and information sharing under supply chain competition," Management Science, vol. 54, no. 4, pp. 701-715, 2008.

[31] B.-X. Li, Y.-W. Zhou, J.-z. Li, and S.-p. Zhou, "Contract choice game of supply chain competition at both manufacturer and retailer levels," International Journal of Production Economics, vol. 143, no. 1, pp. 188-197, 2013.

[32] C. A. Ingene and M. E. Parry, "Bilateral monopoly, identical distributors, and game-theoretic analyses of distribution channels," Journal of the Academy of Marketing Science, vol. 35, no. 4, pp. 586-602, 2007.

[33] S. C. Choi and A. T. Coughlan, "Private label positioning: quality versus feature differentiation from the national brand," Journal of Retailing, vol. 82, no. 2, pp. 79-93, 2006.

[34] G. Cai, Y. Dai, and S. X. Zhou, "Exclusive channels and revenue sharing in a complementary goods market," Marketing Science, vol. 31, no. 1, pp. 172-187, 2012.

[35] J. Chen, L. Liang, D.-Q. Yao, and S. Sun, "Price and quality decisions in dual-channel supply chains," European Journal of Operational Research, vol. 259, no. 3, pp. 935-948, 2017.

[36] C. A. Ingene and M. E. Parry, Mathematical Models of Distribution Channels, Springer, New York, NY, USA, 2004.

[37] B. Liu, G. G. Cai, and A. A. Tsay, "Advertising in asymmetric competing supply chains," Production and Operations Management, vol. 23, no. 11, pp. 1845-1858, 2014.

[38] X. Chen, X. Wang, and X. Jiang, "The impact of power structure on the retail service supply chain with an $\mathrm{O} 2 \mathrm{O}$ mixed channel," Journal of the Operational Research Society, vol. 67, no. 2, pp. 294-301, 2016.

[39] X. Yue and J. Liu, "Demand forecast sharing in a dual-channel supply chain," European Journal of Operational Research, vol. 174, no. 1, pp. 646-667, 2006.

[40] S. Karray, G. Martín-Herrán, and S.-P. Sigué, "Cooperative advertising for competing manufacturers: the impact of longterm promotional effects," International Journal of Production Economics, vol. 184, pp. 21-32, 2017.

[41] S. C. Choi, "Price competition in a duopoly common retailer channel," Journal of Retailing, vol. 72, no. 2, pp. 117-134, 1996.

[42] T. Xiao, T.-M. Choi, and T. C. E. Cheng, "Product variety and channel structure strategy for a retailer-stackelberg supply chain," European Journal of Operational Research, vol. 233, no. 1, pp. 114-124, 2014.

[43] R. Amir and A. Stepanova, "Second-mover advantage and price leadership in bertrand duopoly," Games \& Economic Behavior, vol. 55, no. 1, pp. 1-20, 2000.

[44] S. Choi and K. Fredj, "Price competition and store competition: store brands vs. national brand," European Journal of Operational Research, vol. 225, no. 1, pp. 166-178, 2013.

[45] G. Cai, "Channel selection and coordination in dual-channel supply chains," Journal of Retailing, vol. 86, no. 1, pp. 22-36, 2010.

[46] A. Y. Ha, W. Shang, and Y. Wang, "Manufacturer rebate competition in a supply chain with a common retailer," Production and Operations Management, vol. 26, no. 11, pp. 2122-2136, 2017. 